\title{
On half-homogeneous hyperbolic manifolds and Siegel domains
}

\author{
By \\ Kazufumi NAKAJIMA \\ (Received October 5, 1982)
}

\section{Introduction.}

Siegel domains of the second kind due to Pyatetski-Shapiro [11] are interesting objects of research not only in complex analysis but also in differential geometry. It would be desirable to characterize complex manifolds which are holomorphically isomorphic to Siegel domains among bounded domains in $\boldsymbol{C}^{n}$ or among hyperbolic manifolds. The present paper is an approach to this problem.

Let $M$ be a hyperbolic manifold due to Kobayashi [3] and let $g(M)$ be the Lie algebra of $G(M)$, the identity component of the group of all holomorphic transformation of $M$. We say that $M$ is half-homogeneous if $g(M)_{c}$ (=the complexification of $g(M)$ ) is "transitive" at every point of $M$ (see, $\S 2$ ). In this paper, we shall study half-homogeneous hyperbolic manifolds satisfying a certain condition $(C)$. Let us denote by $\mathfrak{b}^{0}(p)$ the isotropy subalgebra of $g(M)_{c}$ at a point $p$ of $M$. Then the condition $(C)$ implies the existence of an element of $\mathfrak{b}^{0}(p)$ which is mapped to the identity transformation of $T_{p}(M)$ by the isotropy representation (see, §3). Of course, every Siegel domain of the second kind is half-homogeneous and satisfies the condition $(C)$ ([9]). We also introduce the notion of pseudo-Sigel domains in $\S 5$. Now our main results are stated as follows:

(I) Let $M$ be a half-homogeneous hyperbolic manifold satisfying (C). Then $M$ is holomorphically immersed in a complex vector space as a pseudo-Siegel domain $D$ of the second kind in such a way that $G(M$; acts on $D$ equivariantly (Theorem 7.4).

(II) Let $M$ be a half-homogeneous complete hyperbolic manifold satisfying (C). Assume further that $\mathfrak{b}^{0}(p) \neq \mathfrak{b}^{0}(q)$ if $p \neq q$. Then $M$ is holomorphically equivalent to a Siegel domain of the second kind. Conversely, every Siegel domain of the second kind is a hyperbolic manifold having these properties (Theorem 8.2).

(III) Let $M$ be a homogeneous hyperbolic manifold. Then $M$ is isomophic to a homogeneous bounded domain if and only if $M$ satisfies $(C)$ (Theorem 8.3).

Recently, Kodama and Shima [5] obtained other characterization of homogeneous bounded domains.

We now explain the various sections. In $\S 1$, we construct for a hyperbolic manifold $M$ and for a point $p$ of $M$, a complex submanifold $M(p)$ through $p$ by 
the same methods as in $[10]$ and prove that $M(p)$ is a hermitian symmetric space of the non-compact type. We mention that once this is proved, all results in [9] for bounded domains also hold for hyperbolic manifolds.

In $\S 2$, we recall the $G(M)$-equivariant mapping $\Phi$ of a half-homogeneous hyperbolic manifold $M$ into a certain complex coset space $G_{c} / B$ constructed in [9] and rewrite Theorem 3.3 of [9] in more detail for later use. Here $G_{c}$ denote the adjoint group of $g(M)_{c}$. Under the assumption of the half-homogeneity and the condition $(C), \Phi$ becomes an immersion. Moreover there exists an abelian subspace $\theta^{-1}$ of $\mathrm{g}(M)_{c}$ with $\operatorname{dim}_{c} \theta^{-1}=\operatorname{dim}_{C} M$ and a holomorphic imbedding $h_{1}$ of $\theta^{-1}$ into $G_{c} / B$. We show in $\S 3$ that $\Phi(M)$ is contained in $h_{1}\left(\theta^{-1}\right)$ and hence $h_{1}^{-1} \circ \Phi$ is an immersion of $M$ onto a domain $\mathscr{M}$ of $\theta^{-1}$.

Next in $\S 4$, we construct a fibering of $\mathscr{M}$ with the base space $\mathcal{S}$ isomorphic to $M(p)$. We shall show in $\S 5$ that the fiber $\mathscr{M}_{0}$ is isomorphic to a pseudo-Siegel domain.

Since $\mathcal{S}$ is a hermitian symmetric space of the non-compact type, $\mathcal{S}$ is realized as a symmetric Siegel domain $S$. After some preparations in $\S 6$, we construct in $\S 7$ a pseudo-Siegel domain $D$ in a vector space $\operatorname{Ad}^{-1} \delta^{-1}$ with an imbedding $h_{0}: A d \delta^{-1} \theta^{-1} \rightarrow G_{c} / B_{0}$ in such a way that there exists a canonical fibering: $D \rightarrow S$ and that a fiber $D_{0}$ is isomorphic to $\mathscr{M}_{0}$ under a $G_{c}$-equivariant holomorphic diffeomorphism $\tilde{\delta}: G_{c} / B_{0} \rightarrow G_{c} / B$. Here $\delta$ is an element of $G_{c}$ and $B_{0}=A d \delta^{-1} B$. These being prepared, by taking a subgroup $A(S)$ of $A d G(M)$ which acts on $S$ transitively, we shall see $\tilde{\delta} \circ h_{0}(D)=h_{1}(\mathscr{M})$.

Finally in $\S 8$, we shall give characterizations of Siegel domains and homogeneous bounded domains by using the results in the previous sections.

Throughout this paper, we use the following notations: For a hyperbolic manifold $M, \operatorname{Aut}(M)$ means the Lie group of all holomorphic transformations of $M$ and $g(M)$ means its Lie algebra. For a real vector space or a real Lie algebra $A, A_{c}$ denotes its complexification. For any $z \in A_{c}$, we denote by $\bar{z}, \operatorname{Re} z$ and $\operatorname{Im} z$, the complex conjugate, the real part and the imaginary part of $z$ respectively. Let $W$ be a vector space over $\boldsymbol{K}(\boldsymbol{K}=\boldsymbol{R}$ or $\boldsymbol{C})$. We denote by $G r(W ; r, \boldsymbol{K})$ the grassmann manifold consisting of all $r$-dimensional $\boldsymbol{K}$-subspaces of $W$.

\section{$\S 1$. Hermitian symmetric submanifolds of hyperbolic manifolds.}

Let $M$ be a hyperbolic manifold and let $g(M)$ be the Lie algebra of $\operatorname{Aut}(M)$, the group of all holomorphic transformations of $M$. We denote by $\mathfrak{X}(M)$ the space of all vector fields on $M$. For $X \in g(M), X^{*}$ means the element of $\mathfrak{X}(M)$ generated by $\{\exp t X\}_{t \in R}$. The correspondence : $X \rightarrow X^{*}$ can be naturally extended to a linear mapping of $\mathrm{g}(M)_{c}$ to $\mathfrak{X}(M)$ by setting

$$
(X+\sqrt{ }=1 Y)^{*}=X^{*}+J Y^{*} \quad \text { for } \quad X, Y \in \mathrm{g}(M),
$$

where $J$ denotes the complex structure of $M$. By a result of Kobayashi (Theorem 1.4, Ch. III, [4]), this mapping is injective. Let $p$ be a point of $M$. We define subspaces $\mathfrak{b}^{k}(p)$ of $\mathrm{g}(M)_{c}$ for any integer $k$ by 


$$
\begin{aligned}
& \mathfrak{b}^{k}(p)=\mathfrak{g}(M)_{c}, \quad \text { if } \quad k \leqq-1 \\
& \mathfrak{b}^{k}(p)=\left\{Z \in \mathfrak{g}(M)_{c} ; j_{p}^{k}\left(Z^{*}\right)=0\right\} \quad \text { if } \quad k \geqq 0,
\end{aligned}
$$

where $j_{p}^{k}\left(Z^{*}\right)$ denotes the $k$-jet of the vector field $Z^{*}$ at $p$. We then have

$$
\begin{aligned}
& \mathfrak{b}^{i}(p) \supset \mathfrak{b}^{j}(p) \quad \text { if } \quad i \leqq j, \\
& {\left[\mathfrak{b}^{i}(p), \mathfrak{b}^{j}(p)\right] \subset \mathfrak{b}^{i+j}(p) \quad \text { for } \quad i, j \in \boldsymbol{Z} .}
\end{aligned}
$$

Suppose that all derivatives of a vector field $Z^{*}$ at $p$ are zeros and that $Z_{p}^{*}=0$. Then $Z^{*}=0$. Therefore we have

$$
\bigcap_{k=0}^{\infty} \mathfrak{b}^{k}(p)=0
$$

Let $K_{p}$ be the isotropy subgroup of $\operatorname{Aut}(M)$ at $p$ and let $\mathrm{f}_{p}$ be its Lie algebra. We then have

$$
\mathfrak{b}^{0}(p) \cap \overline{\mathfrak{b}^{0}(p)}=\left(\mathfrak{f}_{p}\right)_{c}
$$

and hence

$$
\left[\mathfrak{b}^{1}(p), \overline{\mathfrak{b}^{1}(p)}\right] \subset\left(\mathfrak{f}_{p}\right)_{c} .
$$

The subalgebra $\mathfrak{h}^{0}(p)$ may be considered as the isotropy subalgebra of $g(M)_{c}$ at the point $p$. Let us denote by $\rho_{p}$ the isotropy representation of $\mathfrak{b}^{n}(p)$, i.e., for every $Z \in \mathfrak{b}^{0}(p), \rho_{p}(Z)$ is an endomorphism of $T_{p}(M)$ defined by

$$
\rho_{p}(Z) v=\left[Z^{*}, \xi\right]_{p} \quad \text { for } \quad v \in T_{p}(M),
$$

where $\xi$ is a vector field such that $\xi_{p}=v$. Then an element $Z$ of $\mathfrak{h}^{0}(p)$ belongs to $\mathfrak{b}^{1}(p)$ if and only if $\rho_{p}(Z)=0$. Define a subspace $\mathrm{m}(p)$ by

$$
\mathrm{m}(p)=\left\{Z+\bar{Z} ; Z \in \mathfrak{b}^{1}(p)\right\} .
$$

Clearly $m(p)$ is $A d K_{p}$-invariant.

Lemma 1.1 (cf. [10]). (1) $\mathfrak{f}_{p} \cap \mathrm{m}(p)=0$.

(2) There exists a unique complex structure $I_{p}$ of $m(p)$ such that $\left(I_{p} X\right)^{*}=J X_{p}^{*}$ and the correspondence: $X \rightarrow X+\sqrt{-1} I_{p} X$ gives a linear isomorphism between $\mathrm{m}(p)$ and $\mathfrak{h}^{1}(p)$.

Proof. Let $X \in m(p) \cap \mathfrak{f}_{p}$. There exists $Y \in g(M)$ such that $X+\sqrt{-1} Y$ belongs to $\mathfrak{b}^{1}(p)$. Then $\rho_{p}(X+\sqrt{-1} Y)=0$. It should be noted that $Y$ also belongs to $\mathfrak{m}(p) \cap \mathfrak{f}_{p}$. Since $K_{p}$ is compact, there exists a hermitian inner product $g$ of $T_{p}(M)$ such that both $\rho_{p}(X)$ and $\rho_{p}(Y)$ are skew-symmetric with respect to $g$. For any $v \in T_{p}(M)$, there exists a local vector field $\xi$ around $p$ satisfying $\xi_{p}=v$ and $\mathcal{L}_{\xi} J=0$. We then have $\rho_{p}(\sqrt{-1} Y) v=\left[J Y^{*}, \xi\right]_{p}=J\left[Y^{*}, \xi\right]_{p}=\left[Y^{*}, J \xi\right]_{p}$. Therefore $\rho_{p}(\sqrt{-1} Y)=J \circ \rho_{p}(Y)=\rho_{p}(Y) \circ J$. It follows that $\rho_{p}(\sqrt{-1} Y)$ is symmetric with respect to $g$ and hence $\rho_{p}(X)=\rho_{p}(Y)=0$. This implies $X=Y=0$. The second assertion can be verified by the same way as in [10]. q.e.d. 
We set

$$
\mathfrak{l}_{p}=\mathfrak{f}_{p}+\mathfrak{m}(p) .
$$

Using Lemma 1.1, we get from (1.4)

$$
[X, Y]+\left[I_{p} X, I_{p} Y\right] \equiv 0 \quad\left(\bmod \mathfrak{f}_{p}\right) \quad \text { for } \quad X, Y \in \mathfrak{m}(p) .
$$

On the other hand, since $\left[\mathfrak{b}^{1}(p), \mathfrak{b}^{1}(p)\right] \subset \mathfrak{b}^{1}(p)$, we get

$$
\begin{aligned}
& {[X, Y]-\left[I_{p} X, I_{p} Y\right] \equiv 0 \quad(\bmod \mathfrak{m}(p)) \quad \text { for } X, Y \in \mathfrak{m}(p),} \\
& I_{p}\left([X, Y]-\left[I_{p} X, I_{p} Y\right]\right)=\left[I_{p} X, Y\right]+\left[X, I_{p} Y\right] \quad \text { for } X, Y \in \mathfrak{m}(p) .
\end{aligned}
$$

From (1.5) and (1.6), we have $[\mathfrak{m}(p), \mathfrak{m}(p)] \subset \mathfrak{l}_{p}$ and hence $\mathfrak{l}_{p}$ is a subalgebra of $\mathrm{g}(M)$. Let $L_{p}$ be the connected subgroup of $\operatorname{Aut}(M)$ corresponding to $\mathfrak{I}_{p}$ and put

$$
M(p)=L_{p} \cdot p .
$$

By Lemma 1.1 and (1.7), $M(p)$ is a complex submanifold of $M$ with $\operatorname{dim}_{C} M(p)$ $=\operatorname{dim}_{C} \mathfrak{b}^{1}(p)$.

Let $Z \in \mathfrak{b}^{2}(p)$. By using Lemma 1.1 , we can write $Z=X+\sqrt{-1} I_{p} X$, where $X \in \mathfrak{m}(p)$. Then $[\bar{Z}, Z]=2 \sqrt{-1}\left[I_{p} X, X\right]$. From (1.2), (1.4) and from Lemma 1.1 we have $\left[\mathfrak{b}^{2}(p), \overline{\mathfrak{b}^{2}(p)}\right] \subset\left(\mathfrak{f}_{p}\right)_{c} \cap \mathfrak{b}^{1}(p)=0$. Therefore $\left[I_{p} X, X\right]=0$ and hence $\boldsymbol{R} X$ $+\boldsymbol{R} I_{p} X$ is a complex abelian subalgebra of $g(M)$. Let $L^{\prime}$ be the connected subgroup of $\operatorname{Aut}(M)$ corresponding to $\boldsymbol{R} X+\boldsymbol{R} I_{p} X$. Then the orbite $L^{\prime} \cdot p$ is a complex submanifold of $M$ with a trivial Kobayashi-distance. This means that $L^{\prime} \cdot p=p$ and hence $X \in \mathfrak{f}_{p}$. Thus we get $X=0$ and $\mathfrak{b}^{2}(p)=0$. Consequently, $\left[\mathfrak{b}^{1}(p), \mathfrak{b}^{1}(p)\right]=0$. We then have for any $X, Y \in \mathfrak{m}(p),[X, Y]-\left[I_{p} X, I_{p} Y\right]=0$. This combined with (1.5) tells us

$$
[\mathfrak{m}(p), \mathfrak{m}(p)] \subset \mathfrak{f}_{p} .
$$

Now we can show by the same arguments as in [10] that $M(p)$ is a hermitian symmetric space. Since there is no holomorphic mapping of $C$ into $M(p)$ except constant mappings, every component of $M(p)$ is of the non-compact type. Let us set

$$
\begin{aligned}
& \mathfrak{f}(p)=[\mathfrak{m}(p), \mathfrak{m}(p)] \\
& \mathfrak{l}(p)=\mathfrak{f}(p)+\mathfrak{m}(p) .
\end{aligned}
$$

we can show $\mathfrak{l}(p) \cong \mathfrak{g}(M(p))$ by the same way as in the proof of Proposition 1.1 of [9]. We thereby obtain the following

Theorem 1.2. Let $M$ be a hyperbolic manifold. Then

(1) $\mathfrak{b}^{2}(p)=0$.

(2) $M(p)$ is a hermitian symmetric space of the non-compact type and the subalgebra $\mathfrak{l}(p)$ may be identified with $\mathfrak{g}(M(p))$. In particular, $\mathfrak{r}(p)$ is semi-simple.

Remark 1. For any hermitian manifold $M$, we can construct a complex submanifold $M(p)$ by the same way, changing $\operatorname{Aut}(M)$ to the group of all holomorphic isometries. If $M$ is a Kähler manifold, then we can show that 
$[\mathfrak{m}(p), \mathfrak{m}(p)] \subset \mathfrak{f}_{p}$ and hence $M(p)$ is a hermitian symmetric space ([10]). But this does not hold for a general hermitian manifold. Indeed, let $M$ be a connected complex Lie group with a Lie algebra $m$. With respect to any left invariant hermitian metric, $M$ is a hermitian manifold satisfying $\operatorname{dim}_{C} \mathfrak{b}^{1}(p)=$ $\operatorname{dim}_{C} M$. Moreover $\mathfrak{n}(p)=\mathfrak{m}$ and $M(p)=M$. Therefore if the complex Lie group $M$ admits a left invariant Kähler metric, then $M$ must be abelian because $[\mathfrak{m}, \mathfrak{m}] \subset \mathfrak{f}_{p} \cap \mathfrak{m}=0$. As a consequence, for any non-abelian complex Lie group $M$ equipped with a left invariant hermitian metric, $M(p)(=M)$ is not a hermitian symmetric space.

Remark 2. By virture of Theorem 1.2, all results in $\S \S 2 \sim 5$ of [9] for bounded domains also hold for hyperbolic manifolds. We shall frequently use these results in the following sections.

\section{$\S 2$. An equivariant holomorphic mapping $\Phi$.}

Definition. A hyperbolic manifold $M$ is called half-homogeneous, if for each point $p, T_{p}(M)=\left\{Z_{p}^{*} ; Z \in g(M)_{c}\right\}$.

Let $M$ be a half-homogeneous hyperbolic manifold. From the assumption, $\operatorname{dim}_{C} \mathfrak{h}^{1}(p)$ is constant, say $r_{\| 1}$. Since $T_{p}(M) \cong g(M)_{c} / \mathfrak{b}^{n}(p)$, we have

$$
\mathfrak{b}^{1}(p)=\left\{Z \in \mathfrak{b}^{0}(p) ;\left[Z, \mathfrak{g}(M)_{c}\right] \subset \mathfrak{b}^{0}(p)\right\} .
$$

We define a mapping $\Phi$ of $M$ into the grassmann manifold $\operatorname{Gr}\left(\mathrm{g}(M)_{c} ; r_{0}, C\right)$ by

$$
\Phi(p)=\mathfrak{b}^{0}(p) \quad \text { for } \quad p \in M .
$$

Let $G_{c}$ be the adjoint group of $g(M)_{c}$. The group $G_{c}$ acts on $G r\left(g(M)_{c} ; r_{0}, C\right)$ in a natural manner and $\operatorname{Aut}(M)$ also acts on $\operatorname{Gr}\left(\mathrm{g}(M)_{c} ; r_{0}, C\right)$ by the adjoint representation. It is clear that if $a \in \operatorname{Aut}(M)$ and $p \in M$, then $\Phi(a \cdot p)=A d a \cdot \Phi(p)$. Moreover let $q=\operatorname{Exp} t_{0} Z *(p)$, where $\operatorname{Exp} t Z^{*}$ denotes the one parameter group of local transformations of $M$ generated by $Z^{*}$. Then $\mathfrak{b}^{0}(q)=\exp \left(a d t_{0} Z\right) \mathfrak{b}^{0}(p)$ and hence $\exp \left(a d t_{0} Z\right) \cdot \Phi(p)=\Phi(q)$. It is easy to see that $\Phi$ is holomorphic. In fact, there exist $Z_{1}, \cdots, Z_{n}$ of $\mathrm{g}(M)_{c}$ such that $\left\{\left(Z_{1}\right)_{p}^{*}, \cdots,\left(Z_{n}\right)_{p}^{*}\right\}$ forms a base of $T_{p}(M)$. Then there exist a neighbourhood $W$ of 0 in $C^{n}$ and a neighbourhood $U$ of $p$ such that the following mapping $g_{1}$ is a holomorphic diffeomorphism of $W$ onto $U$;

$$
\begin{gathered}
g_{1}\left(z_{1}, \cdots, z_{n}\right)=\operatorname{Exp} x_{1} Z_{1}^{*} \circ \operatorname{Exp} y_{1} J Z_{1}^{*} \cdots \cdots \\
z_{i}=x_{i}+\sqrt{-1} y_{n} .
\end{gathered}
$$

Let $g_{2}$ be a holomorphic mapping of $W$ to $G_{c}$ defined by

$$
g_{2}\left(z_{1}, \cdots, z_{n}\right)=\exp \left(\text { ad } z_{1} Z_{1}\right) \cdots \cdot \exp \left(\text { ad } z_{n} Z_{n}\right) .
$$

We then have $\Phi(q)=g_{2} \circ g_{1}^{-1}(q) \cdot \Phi(p)$ for $q \in U$. Therefore $\Phi$ is holomorphic.

By Theorem 1.2, $\mathfrak{l}(p)$ is a semi-simple Lie algebra corresponding to the 
hermitian symmetric space $M(p)$ of the non-compact type. Therefore there exists a unique $Z_{p}$ of $\mathfrak{f}(p)$ such that

$$
\begin{array}{lll}
\text { ad } Z_{p} X=I_{p} X & \text { for } & X \in \mathfrak{m}(p), \\
\text { ad } Z_{p} Y=0 & \text { for } & Y \in \mathfrak{F}(p) .
\end{array}
$$

From Theorem 3.3 of [9], we know that for any $p, q \in M$, there exists $f$ of $G_{c}$ such that $f \cdot \Phi(p)=\Phi(q)$ and $f(\mathfrak{l}(p))=\mathfrak{l}(q)$. We recall its proof and verify the following

Lemma 2.1. Let $M$ be a half-homogeneous hyperbolic manifold and let $p, q$ be two points of $M$. Then there exist contineous mappings $c_{t}:[0,1] \rightarrow M$ and $f_{t}:[0,1] \rightarrow G_{c}$ satisfying

(a) $c_{0}=p, c_{1}=q$ and $f_{0}=1$.

(b) $f_{t} \cdot \Phi(p)=\Phi\left(c_{t}\right)$ and $f_{t}(\mathfrak{l}(p))=\mathfrak{l}\left(c_{t}\right)$.

Let $p, q \in M$. There exist $X_{1}, \cdots, X_{m} \in \mathfrak{g}(M)_{c}$ and $p_{0}, \cdots, p_{m} \in M$ such that $p_{0}=p, p_{m}=q$ and $p_{i}=\operatorname{Exp} X_{i}^{*}\left(p_{i-1}\right)$ for $i \geqq 1$. We put for $\frac{i}{m} \leqq t \leqq \frac{i+1}{m}$

$$
\begin{aligned}
& c_{t}=\operatorname{Exp}(t m-i) X_{i+1}^{*}\left(p_{i}\right), \\
& g_{t}=\exp \left(a d(t m-i) X_{i+1}\right) \cdot \exp \left(\operatorname{ad} X_{i}\right) \cdots \cdot \exp \left(\text { ad } X_{1}\right) .
\end{aligned}
$$

It is clear that $\left(c_{t}, g_{t}\right)$ satisfies the properties of the lemma except the equation $g_{t}(\mathfrak{l}(p))=\mathfrak{l}\left(c_{t}\right)$. Using (2.1), we get $g_{t} \cdot \mathfrak{b}^{1}(p)=\mathfrak{b}^{1}\left(c_{t}\right)$. We set $E_{t}=\sqrt{-1} Z_{c_{t}}$ and $A_{\lambda}=\left\{X \in \mathfrak{g}(M)_{c} ;\left[E_{0}, X\right]=\lambda X\right\}$ for $\lambda \in \boldsymbol{R}$. By Lemma 3.4 of $[9], \mathrm{g}(M)=\sum_{-1 ; \lambda=1} A_{\lambda}$ and as in the proof of Theorem 3.3 of [9], we get

$$
g_{t}^{-1} E_{t}=E_{0}+\sum_{0<\lambda \leq 1} a_{\lambda}(t), \quad a_{\lambda}(t) \in A_{\lambda} .
$$

Let $\lambda_{1}$ denote the smallest positive number $\lambda$ satisfying $A_{\lambda} \neq 0$. By a direct computation, we have

$$
\exp \left(\frac{1}{\lambda_{1}} a d a_{\lambda_{1}}(t)\right) \cdot g_{t}^{-1} \cdot E_{t}=E_{0}+\sum_{\lambda_{1}<\lambda \leq 1} a_{\lambda}^{\prime}(t), \quad a_{\lambda}^{\prime}(t) \in A_{\lambda} .
$$

Since $A_{\lambda} \subset \mathfrak{b}^{0}(p)$ for $\lambda>0$ (Lemma 3.4, [9]), repeating this process, we obtain $h_{t} \in G_{c}$ such that $h_{0}=1$ and

$$
h_{t} \cdot g_{t}^{-1} \cdot E_{t}=E_{0} \text { and } h_{t} \cdot g_{t}^{-1} \cdot \mathfrak{b}^{0}\left(c_{t}\right)=\mathfrak{b}^{0}(p) .
$$

Notice that if $E_{t}$ depends contineously on $t$, then so does $h_{t}$. From $(b)$ of Lemma 3.4 of [9] and (2.3), we have

$$
h_{t} \cdot g_{t}^{-1} \cdot \mathfrak{b}^{1}\left(c_{t}\right)=\mathfrak{b}^{1}(p) \text { and } h_{t} \cdot g_{t}^{-1} \cdot \overline{\mathfrak{b}^{1}\left(c_{t}\right)}=\overline{\mathfrak{b}^{1}(p)} \text {. }
$$

Hence we get

$$
h_{t} \cdot g_{t}^{-1} \cdot \mathfrak{f}\left(c_{t}\right)_{c}=\mathfrak{F}(p)_{c} \text { and } h_{t} \cdot g_{t}^{-1} \cdot \mathfrak{m}\left(c_{t}\right)_{c}=\mathfrak{m}(p)_{c} \text {. }
$$

We set $\mathfrak{l l}(t)=h_{t} \cdot g_{t}^{-1}\left(\mathfrak{f}\left(c_{t}\right)+\sqrt{-1} \mathfrak{m}\left(c_{t}\right)\right)$. Then $\mathfrak{n}(t)$ is a compact real form of $\mathfrak{l}(p)_{c}$ 
for any $t$. Let $\sigma_{t}$ be the conjugation of $\mathfrak{l}(p)_{c}$ with respect to $\mathfrak{u}(t)$. Then $\sigma_{t} \circ \sigma_{0}$ is an automorphism of $\mathfrak{I}(p)_{c}$. Let $\alpha_{0}$ be the positive definite hermitian form on $\mathfrak{I}(p)_{c}$ defined by $\alpha_{0}(X, Y)=-\alpha\left(X, \sigma_{0} Y\right)$ for $X, Y \in \mathfrak{I}(p)_{c}$, where $\alpha$ denotes the killing form of $\mathfrak{l}(p)_{c}$. By a direct calculation, we know that $\sigma_{t}{ }^{\circ} \sigma_{0}$ is hermitian symmetric with respect to $\alpha_{0}$. Recall that the space of all hermitian symmetric endomorphisms is diffeomorphic to that of all hermitian symmetric linear transformations with positive eigenvalues under the exponential mapping. Therefore there corresponds a hermitian symmetric endomorphism $H(t)$ of $\mathfrak{I}(p)_{c}$ such that $\exp H(t)=\left(\sigma_{t} \circ \sigma_{0}\right)^{2}$. We set $P_{t}^{s}=\exp s H(t)$. In view of the proof of Theorem 7.2 in Ch. III of [1], we know

$$
P_{t}^{1 / 4} \mathfrak{l}(0)=\mathfrak{l}(t) .
$$

Moreover for any $t,\left\{P_{t}^{s}\right\}_{s \in R}$ is a one parameter subgroup of automorphisms of the semi-simple Lie algebra $\mathfrak{l}(p)_{c}$. Hence there exists uniquely $X(t) \in \mathfrak{l}(p)_{c}$ such that $H(t)=a d X(t)$. Since $E_{0} \in \sqrt{-1} \mathfrak{u}(t), \sigma_{t} \circ \sigma_{0}\left(E_{0}\right)=E_{0}$. This means $P_{t}^{s} E_{0}=E_{0}$ and hence $\left[X(t), E_{0}\right]=0$. Form this equation, we get $X(t) \in \mathfrak{l}(p)_{c}$. We now set

$$
f_{t}=g_{t} \cdot h_{t}^{-1} \cdot \exp \left(\frac{1}{4} \text { ad } X(t)\right) \text {. }
$$

Clearly $f_{t} \mathfrak{b}^{0}(p)=\mathfrak{b}^{0}\left(c_{t}\right)$. By (2.4) and (2.5), we have

$$
\begin{aligned}
& f_{t} \cdot \mathfrak{f}(p) \subset\left(g_{t} \cdot h_{t}^{-1} \cdot \mathfrak{l}(t)\right) \cap \mathfrak{f}\left(c_{t}\right)_{c}=\mathfrak{t}\left(c_{t}\right), \\
& f_{t} \cdot \mathfrak{m}(p) \subset\left(g_{\iota} \cdot h_{t}^{-1} \cdot \sqrt{-1} \mathfrak{l}(t)\right) \cap \mathfrak{m}\left(c_{t}\right)_{c}=\mathfrak{m}\left(c_{t}\right) .
\end{aligned}
$$

We also know from (2.4), $\operatorname{dim}_{R} \mathfrak{f}\left(c_{t}\right)=\operatorname{dim}_{R} \mathfrak{f}(p)$ and $\operatorname{dim}_{R} \mathfrak{m}\left(c_{t}\right)=\operatorname{dim}_{R} \mathfrak{m}(p)$. Therefore we get $f_{t} \cdot \mathfrak{f}(p)=\mathfrak{f}\left(c_{t}\right)$ and $f_{t} \cdot \mathfrak{m}(p)=\mathfrak{m}\left(c_{t}\right)$. We thereby proved Lemma 2.1 except showing that $f_{t}$ is contineous. We have already proved that for any $p, q \in M$, $\mathfrak{I}(p)$ and $\mathfrak{I}(q)$ are isomorphic to each other. In particular $\operatorname{dim}_{R} \mathfrak{m}(p)$ and $\operatorname{dim}_{R}^{\mathfrak{f}}(p)$ are constant. To complete the proof of Lemma 2.1, it is sufficient to show the following

Proposition 2.2. (1) The correspondences: $p \rightarrow \mathfrak{m}(p)$ and $p \rightarrow \mathfrak{P}(p)$ are differentiable mappings of $M$ into $G r\left(g(M) ; r_{m}, \boldsymbol{R}\right)$ and into $G r\left(g(M) ; r_{k}, \boldsymbol{R}\right)$ respectively, where $r_{m}=\operatorname{dim}_{R} \mathfrak{m}(p)$ and $r_{k}=\operatorname{dim}_{R} \mathfrak{k}(p)$.

(2) The correspondence $p \rightarrow Z_{p}$ is a differentiable mapping of $M$ into $\mathfrak{g}(M)$, where $Z_{p}$ is the element of $\mathfrak{f}(p)$ defined by (2.2).

Proof. We recall that if $f \in G_{c}$ and $f \cdot \mathfrak{b}^{0}(p)=\mathfrak{b}^{0}(q)$, then $f \cdot \mathfrak{b}^{1}(p)=\mathfrak{b}^{1}(q)$ because of (2.1). Therefore we can show by the same way as in the case of the mapping $\Phi$, that the assignment: $p \rightarrow \mathfrak{b}^{1}(p)$ is holomorphic. Since $\operatorname{dim}_{C} \mathfrak{b}^{1}(p)=\frac{1}{2} \operatorname{dim}_{R} \mathfrak{m}(p)$, we can take for any point $p$, locally defined differentiable mappings $w_{1}\left(p^{\prime}\right), \cdots, w_{r_{m} / 2}\left(p^{\prime}\right)$ of a neighbourhood of $p$ into $g(M)_{c}$ in such a way that $\left\{w_{1}\left(p^{\prime}\right), \cdots, w_{r_{m} / 2}\left(p^{\prime}\right)\right\}$ forms a base of $\mathfrak{b}^{1}\left(p^{\prime}\right)$. We write $w_{1}\left(p^{\prime}\right)=u_{i}\left(p^{\prime}\right)+\sqrt{-1} v_{i}\left(p^{\prime}\right)$, where $u_{i}\left(p^{\prime}\right), v_{i}\left(p^{\prime}\right) \in \mathfrak{m}\left(p^{\prime}\right)$. Then the set $\left\{u_{i}\left(p^{\prime}\right), v_{j}\left(p^{\prime}\right)\right\}_{1 \leq i, j \leq r_{m} / 2}$ forms a base of $\mathfrak{m}\left(p^{\prime}\right)$. Since $\mathfrak{f}\left(p^{\prime}\right)=\left[\mathfrak{m}\left(p^{\prime}\right), \mathfrak{m}\left(p^{\prime}\right)\right]$ and since $\operatorname{dim}_{\boldsymbol{R}} \mathfrak{f}\left(p^{\prime}\right)$ is constant, we can take 
a base $\left\{e_{1}\left(p^{\prime}\right), \cdots, e_{r_{k}}\left(p^{\prime}\right)\right\}$ of $\mathfrak{f}^{\prime}\left(p^{\prime}\right)$ in such a way that $e_{i}\left(p^{\prime}\right)$ depends also differentiably on $p^{\prime}$. Thus we get (1).

By using a base $\left\{u_{i}\left(p^{\prime}\right), v_{j}\left(p^{\prime}\right)\right\}_{1 \leq i, j \leq r_{m} / 2}, I_{p^{\prime}}$ is represented by a matrix $Q$ which is independent to $p^{\prime}$. Let us write $Z_{p^{\prime}}=\sum_{i} \nu_{i}\left(p^{\prime}\right) e_{i}\left(p^{\prime}\right)$. As an endomorphism of $\mathfrak{m}\left(p^{\prime}\right)$, each $a d e_{i}\left(p^{\prime}\right)$ is represented by a matrix $Q_{i}\left(p^{\prime}\right)$ with respect to a base $\left\{u_{i}\left(p^{\prime}\right), v_{j}\left(p^{\prime}\right)\right\}_{1 \leq i, j \leq r_{m} / 2}$. Then $Q_{1}\left(p^{\prime}\right), \cdots, Q_{r_{k}}\left(p^{\prime}\right)$ are linearly independent. Now $\left\{\nu_{1}\left(p^{\prime}\right), \cdots, \nu_{r_{k}}\left(p^{\prime}\right)\right\}$ is a unique solution of the equation $\sum_{i} \nu_{i}\left(p^{\prime}\right) Q_{i}\left(p^{\prime}\right)=Q$.

This implies that $\nu_{i}\left(p^{\prime}\right)$ is differentiable and we get (2).

q.e.d.

\section{§3. A holomorphic immersion.}

Let $M$ be a half-homogeneous hyperbolic manifold and let $\rho_{p}$ be the isotropy representation of $\mathfrak{b}^{0}(p)$. We consider the following condition:

(C) There exists $H_{p}$ of $\mathfrak{b}^{0}(p)$ such that $\rho_{p}\left(H_{p}\right)=1$.

By the half-homogeneity, if $M$ satisfies $(C)$ at a point $p$, then $M$ satisfies at any point of $M$. In what follows, we fix a point $p$ of $M$ and assume that $M$ satisfies (C). Put

$$
\theta^{\lambda}=\left\{X \in g(M)_{c} ;\left[H_{p}, X\right]=\lambda X\right\} .
$$

We then have (Lemma 4.1, [9])

$$
\begin{aligned}
& g(M)_{c}=\theta^{-1}+\theta^{0}+\theta^{1}, \quad\left[\theta^{\lambda}, \theta^{2}\right] \subset \theta^{\lambda+\nu}, \\
& \mathfrak{b}^{0}(p)=\theta^{0}+\theta^{1}, \\
& \mathfrak{b}^{1}(p)=\theta^{1} .
\end{aligned}
$$

Since $M$ is half-homogeneous, we have $\operatorname{dim}_{C} \theta^{-1}=\operatorname{dim}_{C} M$. Moreover we may assume (Lemma 4.2, [9])

$$
\overline{\mathfrak{b}^{1}(p)} \subset \theta^{-1} .
$$

Let $\mathfrak{r}$ denotes the radical of $\mathfrak{g}(M)$ and put

$$
\mathfrak{t}=[\mathfrak{r}, \mathfrak{l}(p)] .
$$

We also define a subalgebra $a$ of $g(M)$ by

$$
\mathfrak{a}=\{X \in \mathfrak{g}(M) ;[X, \mathfrak{l}(p)]=0\} .
$$

If we put

$$
\mathfrak{l}_{+}=\overline{\mathfrak{b}^{1}(p)}, \quad \mathfrak{l}_{-}=\mathfrak{b}^{1}(p) \text { and } \quad E_{p}=\sqrt{-1} Z_{p},
$$

we then have from Proposition 2.4 of [9],

$$
\begin{aligned}
& \mathfrak{g}(M)=\mathfrak{l}(p)+\mathfrak{t}+\mathfrak{a} \quad(\text { vector } \\
& \theta^{-1}=\mathfrak{l}_{+}+\mathfrak{t}_{c} \cap \theta^{-1}+\mathfrak{a}_{c} \cap \theta^{-1} \quad \text { (vector space direct sum) } \\
& \theta^{0}=\mathfrak{f}(p)_{c}+\mathfrak{t}_{c} \cap \theta^{0}+\mathfrak{a}_{c} \cap \theta^{0} \quad \text { (vector space direct sum) } \\
& \mathfrak{t}_{c}=\mathfrak{t}_{c} \cap \theta^{-1}+\mathfrak{t}_{c} \cap \theta^{0} \\
& \mathfrak{a}_{c}=\mathfrak{a}_{c} \cap \theta^{-1}+\mathfrak{a}_{c} \cap \theta^{0}
\end{aligned}
$$


and

$$
\begin{aligned}
& \mathfrak{r}_{c}=\sum_{-1<\lambda<1}\left(\mathfrak{r}_{c}\right)_{\lambda} \\
& \mathfrak{t}_{c} \cap \theta^{-1}=\sum_{-1<\lambda<0}\left(\mathfrak{r}_{c}\right)_{\lambda}, \quad \mathfrak{t}_{c} \cap \theta^{0}=\sum_{0<\lambda<1}\left(\mathfrak{r}_{c}\right)_{\lambda} \\
& \mathfrak{a}_{c} \cap \theta^{-1}=\left(\mathfrak{r}_{c}\right)_{0} \cap \theta^{-1},
\end{aligned}
$$

where $\left(\mathfrak{r}_{c}\right)_{\lambda}=\left\{X \in \mathfrak{r}_{c} ;\left[E_{p}, X\right]=\lambda X\right\}$.

Let $B$ be the subgroup of the adjoint group $G_{c}$ defined by

$$
B=\left\{f \in G_{c} ; f \cdot \mathfrak{b}^{0}(p)=\mathfrak{b}^{0}(p)\right\} .
$$

Since $g(M)_{c}$ is centerless (Lemma 5.1, [9]), the Lie algebra of $G_{c}$ is identified with $g(M)_{c}$. It is easy to see that under this identification, the Lie algebra of $B$ coincides with $\mathfrak{b}^{0}(p)$. The homogeneous space $G_{c} / B$ is the $G_{c}$-orbit of $\mathfrak{b}^{0}(p)$ in $\operatorname{Gr}\left(\mathrm{g}(M)_{c} ; r_{0}, C\right)$. Let $\Phi$ be the holomorphic mapping defined in $\S 2$. Then $\Phi$ is an immersion of $M$ onto an open subset of $G_{c} / B$ (Propositions 3.1 and 5.2, [9]). Let $h_{1}$ be the holomorphic mapping of $\theta^{-1}$ to $G_{c} / B$ defined by

$$
h_{1}(z)=\pi_{1} \cdot \exp z \quad \text { for } \quad z \in \theta^{-1},
$$

where $\pi_{1}$ denotes the projection of $G_{c}$ onto $G_{c} / B$. It is easy to see that $h_{1}$ is a holomorphic imbedding of $\theta^{-1}$ onto an open dense subset of $G_{c} / B$ (cf. Proof of Theorem 1, [8]).

Lemma 3.1. Let $X_{-1} \in \theta^{-1}$ and $X_{0} \in \theta^{0}$. Then $\exp X_{-1}$ (resp. exp $X_{0}$ ) leaves $h_{1}\left(\theta^{-1}\right)$ invariant and induces a translation (resp. a linear transformation) of $\theta^{-1}$.

Proof. For any $z \in \theta^{-1}$, we get

$$
\begin{aligned}
& \exp X_{-1} \cdot h_{1}(z)=\pi_{1}\left(\exp X_{-1} \cdot \exp z\right)=h_{1}\left(X_{-1}+z\right) \\
& \begin{aligned}
\exp X_{0} \cdot h_{1}(z) & =\pi_{1}\left(\exp X_{0} \cdot \exp z \cdot\left(\exp X_{0}\right)^{-1} \cdot \exp X_{0}\right) \\
& =h_{1}\left(\operatorname{Ad}\left(\exp X_{0}\right) z\right) .
\end{aligned}
\end{aligned}
$$

Let $L, K, L_{c}, K_{c}, L_{+}$and $L_{-}$be the connected subgroup of $G_{c}$ corresponding to the subalgebra $\mathfrak{I}(p), \mathfrak{f}(p), \mathfrak{r}(p)_{c}, \mathfrak{f}(p)_{c}, \mathfrak{I}_{+}$and $\mathfrak{L}_{-}$respectively. Recall that $\Phi$ is an imbedding on $M(p)$ (Proposition 3.2, [9]). Then $\Phi(M(p))=L / K$. It is well known that the mapping:

$$
L_{+} \times K_{c} \times L_{-} \ni(a, b, c) \longrightarrow a \cdot b \cdot c \in L_{c}
$$

is a holomorphic diffeomorphism onto an open set of $L_{c}$ and that

$$
L \subset L_{+} \cdot K_{c} \cdot L_{-} \cdot
$$

Therefore $\Phi(M(p))$ is contained in $h_{1}\left(\mathfrak{l}_{+}\right)$. We set

$$
\mathcal{S}=h_{1}^{-1} \odot \Phi(M(p)) \text {. }
$$

$\mathcal{S}$ is a symmetric bounded domain in $\mathfrak{l}_{+}$and is known as the Harish-Chandra realization of $M(p)$. We now prove the following 
Theorem 3.2. Let $M$ be a half-homogeneous hyperbolic manifold satisfying the condition $(C)$. Then $\Phi(M)$ is contained in $h_{1}\left(\theta^{-1}\right)$ and therefore $h_{1}^{-1} \circ \Phi$ gives a holomorphic immersion of $M$ onto an open set of the vector space $\theta^{-1}$.

Proof. Let $R_{c}$ denotes the connected subgroup of $G_{c}$ corresponding to $(\mathfrak{t}+\mathfrak{a})_{c}\left(=(\mathfrak{r}+\mathfrak{a})_{c}\right)$. Note that $(\mathfrak{t}+\mathfrak{a})_{c}$ is an ideal of $\mathfrak{g}(M)_{c}$. Therefore every element of $G_{c}$ induces an automorphism of $\mathrm{g}(M)_{c} /(\mathfrak{t}+\mathfrak{a})_{c}$. Since $\mathrm{g}(M)_{c}=\mathfrak{l}(p)_{c}+(\mathfrak{t}+\mathfrak{a})_{c}$, the group $R_{c}$ is the identity component of the kernel of this correspondence. Therefore $R_{c}$ is closed. Clearly $G_{c}=R_{c} \cdot L_{c}$.

Let $q$ be any point of $M$ and let $\left(c_{t}, f_{t}\right)$ be as in Lemma 2.1. We denote by $\gamma$ the projection of $G_{c}$ onto $G_{c} / R_{c}$. The restriction of $\gamma$ to $L_{c}$ gives a covering mapping of $L_{c}$ onto $G_{c} / R_{c}$. Therefore there exists a contineous curve $s_{t}$ in $L_{c}$ such that $\gamma\left(s_{t}\right)=\gamma\left(f_{t}\right)$ and $s_{0}=1$. We set $r_{t}=f_{t} \cdot s_{t}^{-1}$. Then $r_{t}$ is a contineous curve contained in $R_{c}$. Clearly

$$
a X \equiv X \quad\left(\bmod (\mathfrak{t}+\mathfrak{a})_{c}\right) \quad \text { for } \quad X \in \mathfrak{l}(p)_{c} \text { and } a \in R_{c} .
$$

It follows that for any $X \in \mathfrak{l}(p)_{c}, f_{t} X \equiv s_{t} X\left(\bmod (t+\mathfrak{a})_{c}\right)$. Since $f_{t} X \in \mathrm{g}(M)$ for any $X \in \mathfrak{I}(p)$, we know

$$
s_{t} X \in \mathfrak{g}(M) \cap \mathfrak{l}(p)_{c}=\mathfrak{l}(p) .
$$

Notice that $L$ is an identity component of the subgroup of $L_{c}$ which consists of all elements of $L_{c}$ leaving $\mathfrak{I}(p)$ invariant. It follows that $s_{t}$ is contained in $L$. Consequently, $s_{1} \cdot \Phi(p)$ is contained in $h_{1}(S)$. Since $(t+\mathfrak{a})_{c}$ is contained in $\theta^{-1}+\theta^{0}$, we get by Lemma 3.1 ,

$$
\Phi(q)=f_{1} \cdot \Phi(p) \in R_{c} \cdot h_{1}(\mathcal{S}) \subset h_{1}\left(\theta^{-1}\right) . \quad \text { q. e. d. }
$$

The next theorem gives a characterization of hermitian symmetric spaces of the non-compact type among half-homogeneous hyperbolic manifolds.

Theorem 3.3. Let $M$ be a half-homogeneous hyperbolic manifold. Then $M$ is a hermitian symmetric space if and only if there exists $H_{p}$ in $\sqrt{-1} t_{p}$ such that $\rho_{p}\left(H_{p}\right)=1$.

Proof. Suppose that $H_{p} \in \sqrt{-1} t_{p}$ satisfying $\rho_{p}\left(H_{p}\right)=1$. Then $\theta^{-1}=\overline{\theta^{1}}$. Therefore $\operatorname{dim}_{c} \theta^{1}=\operatorname{dim}_{C} M$. Since $\theta^{1}=\mathfrak{b}^{1}(p)$, this implies that $M(p)=M$ and hence $M$ is a hermitian symmetric space. The converse is clear. q.e.d.

\section{$\S 4$. Fiberings of $M$ and $h_{1}^{-1} \circ \Phi(M)$.}

Let $M$ be a half-homogeneous hyperbolic manifold satisfying $(C)$. We set

$$
\mathscr{M}=h_{1}^{-1} \circ \Phi(M) \text {. }
$$

Every element of $\operatorname{Aut}(M)$ leaves $\Phi(M)$ invariant and hence induces an automorphism of the domain $\mathscr{M}$. In this section, we study the action of the group $G(M)$, the identity component of $\operatorname{Aut}(M)$, and construct fiberings of $M$ and $\mathscr{M}$ 
with $S$ as base spaces.

For convenience, let us set

$$
\mathrm{t}_{+}=\mathrm{t}_{c} \cap \theta^{-1}, \quad \mathrm{t}_{-}=\mathrm{t}_{c} \cap \theta^{0} \quad \text { and } \quad \mathfrak{u}=\mathrm{a}_{c} / \cap \theta^{-2} .
$$

Since $\overline{\left(\mathfrak{r}_{c}\right)_{\lambda}}=\left(\mathfrak{r}_{c}\right)_{-\lambda}$, we get from (3.3),

$$
\overline{\mathrm{t}_{+}}=\mathrm{t}_{-}
$$

and from Proposition 2.4 of [9],

$$
\mathrm{t}_{+}=\left[\mathrm{t}_{-}, \mathfrak{l}_{+}\right], \mathrm{t}_{-}=\left[\mathrm{t}_{+}, \mathfrak{l}_{-}\right] .
$$

Since $\left[E_{p}, \mathfrak{f}(p)\right]=0$ and since $\mathfrak{r}_{c}$ is contained in $\theta^{-1}+\theta^{0}$, we get from (3.1) and (3.2)

$$
\left[\mathfrak{f}(p)_{c}, \mathrm{t}_{ \pm}\right] \subset \mathrm{t}_{ \pm}, \quad\left[\mathfrak{l}_{+}, \mathrm{t}_{+}\right]=0 \text { and }\left[\mathfrak{l}_{-}, \mathrm{t}_{-}\right]=0 \text {. }
$$

Lemma 4.1. (1) $\left[t_{-}, t_{-}\right]=\left[t_{+}, t_{+}\right]=0$ and $\left[t_{+}, t_{-}\right] \subset u$.

(2) $[t, \mathfrak{u}]=0$.

(3) $[t,[t, t]]=0$.

Proof. If $-1<\lambda<0$ and $0<\nu<1$, then $\left(\mathfrak{r}_{c}\right)_{\lambda} \subset \theta^{-1}$ and $\left(\mathfrak{r}_{c}\right)_{2} \subset \theta^{0}$. Therefore $\left[\left(\mathfrak{r}_{c}\right)_{\lambda},\left(\mathfrak{r}_{c}\right)_{\nu}\right] \subset \theta^{-1} \cap\left(\mathfrak{r}_{c}\right)_{\lambda+\nu}$. On the other hand

$$
\left[\left(\mathfrak{r}_{c}\right)_{\lambda},\left(\mathfrak{r}_{c}\right)_{\nu}\right] \subset\left[\overline{\left(\mathfrak{r}_{c}\right)_{-\lambda}}, \overline{\left(\mathfrak{r}_{c}\right)_{-\nu}}\right] \subset \overline{\left(\mathfrak{r}_{c}\right)_{-(\lambda+\nu)} \cap \theta^{-1}} .
$$

If $\lambda+\nu \neq 0$, then $\overline{\left(\mathfrak{r}_{c}\right)_{-(\lambda+\nu)} \cap \theta^{-1}} \subset \theta^{0}$ by (3.3). It follows that if $\lambda+\nu \neq 0$, then $\left[\left(\mathfrak{r}_{c}\right)_{\lambda},\left(\mathfrak{r}_{c}\right)_{\nu}\right]=0$ and $\left[\left(\mathfrak{r}_{c}\right)_{\lambda},\left(\mathfrak{r}_{c}\right)_{-\lambda}\right] \subset\left(\mathfrak{r}_{c}\right)_{0} \cap \theta^{-1}$. Therefore $\left[\mathfrak{t}_{+}, \mathfrak{t}_{-}\right] \subset \mathfrak{u}$. If $0<\nu, \mu<1$,

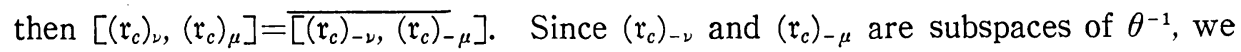
get $\left[\left(\mathfrak{r}_{c}\right)_{\nu},\left(\mathfrak{r}_{c}\right)_{\mu}\right]=0$. This implies $\left[\mathfrak{t}_{-}, \mathfrak{t}_{-}\right]=0$. If $0<\mu<1$, then $\left[\left(\mathfrak{r}_{c}\right)_{\mu}, \mathfrak{u}\right] \subset \theta^{-1} \cap\left(\mathfrak{r}_{c}\right)_{\mu}$ $=0$ by (3.3). Since $\left[t_{+}, t_{+}+\mathfrak{l t}\right]=0$, we get (1) and (2). Consequently,

$$
[\mathrm{t},[\mathrm{t}, \mathrm{t}]] \subset\left[\mathrm{t},\left[\mathrm{t}_{+}, \mathrm{t}_{-}\right]\right] \subset[\mathrm{t}, \mathrm{u}]=0 \text {. }
$$

Hence we get (3).

$$
\text { q.e.d. }
$$

Let us denote by $\eta_{1}$ the projection of $\mathfrak{u} \times t_{+} \times \mathfrak{l}_{+}\left(=\theta^{-1}\right)$ onto $\mathfrak{I}_{+}$. In view of the proof of Theorem 3.2, $\eta_{1} \circ h_{1}^{-1} \circ \Phi(M)=\mathcal{S}$. Therefore the domain $\mathscr{M}$ is an open set of $\mathfrak{u} \times t_{+} \times \mathcal{S}$. Let $g \in L$. Then $g$ induces an automorphism of $\mathcal{S}$ which will be denoted by $g_{s}$. In what follows, for every $w \in t_{c}$, we denote by $w_{+}$and by $w$ - the $t_{+}$- and the $t_{-}$-component of $w$ respectively.

Lemma 4.2. Every $g \in L$ leaves $h_{1}\left(\mathfrak{u} \times t_{+} \times S\right)$ invariant and hence induces a holomorphic transformation $\tilde{g}$ of $\mathfrak{u} \times t_{+} \times \mathcal{S}$. Let $\tilde{g}(u, w, z)=\left(u^{\prime}, w^{\prime}, z^{\prime}\right)$. Then

$$
\begin{aligned}
& z^{\prime}=g_{s}(z), \\
& w^{\prime}=(A d g w)_{+}-\left[z^{\prime},(\text { Ad } g w)_{-}\right], \\
& u^{\prime}=u-\frac{1}{2}\left[w^{\prime}, \text { Ad } g w\right] .
\end{aligned}
$$

Using Lemma 4.1, this lemma can be verified similarly as Lemma 4.5 of [7]. 
Let $R$ denote the connected subgroup of $G_{c}$ corresponding to $t+a$. If $f \in R$, then $f$ induces an affine transformation $\tilde{f}$ of $\mathfrak{u} \times \mathrm{t}_{+} \times \mathfrak{l}_{+}$by Lemma 3.1. If $\tilde{f}(u, w, z)$ $=\left(u^{\prime}, w^{\prime}, z^{\prime}\right)$, then $z=z^{\prime}$ by (3.4). This combined with Lemma 4.2 leads us to the following

Proposition 4.3. Every $g \in G(M)$ induces automorphisms $g_{\mathscr{M}}$ of $\mathscr{M}$ and $g_{S}$ of $\mathcal{S}$ in such a way that the following diagram commutes:

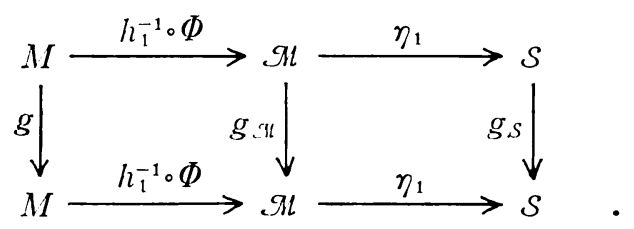

We set for $z \in \mathcal{S}$

$$
M_{z}=\left(\eta_{1} \circ h_{1}^{-1} \circ \Phi\right)^{-1}(z) \text { and } \mathscr{M}_{z}=\eta_{1}^{-1}(z) .
$$

Since $L$ acts on $\mathcal{S}$ transitively, we get from Lemma 4.2 and Proposition 4.3 the following

Proposition 4.4. Let $z, z^{\prime} \in \mathcal{S}$. Then the fibers $M_{z}$ (resp. $\mathscr{M}_{z}$ ) and $M_{z^{\prime}}$ (resp. $\mathscr{M}_{z^{\prime}}$ ) are holomorphically isomorphic to each other.

Remark 3. Both $\left(M, \mathcal{S}, \eta_{1} \circ h_{1}^{-1} \circ \Phi\right)$ and $\left(\mathcal{M}, \mathcal{S}, \eta_{1}\right)$ give fiberings with base space $\mathcal{S}$. These are not holomorphic fiber bundles. But real analytically, we have $M \cong \mathcal{S} \times M_{0}$ and $\mathscr{M} \cong \mathcal{S} \times \mathscr{M}_{0}$. In fact let $q \in M$ and $z=\eta_{1} \circ h_{1}^{-1} \bullet \Phi(q)$. Since the mapping $\beta: X \rightarrow(\exp X)_{\mathcal{S}} \cdot 0$ gives an analytic diffeomorphism of $\mathfrak{m}(p)$ onto $\mathcal{S}$, we get a real analytic diffeomorphism of $M$ onto $\mathcal{S} \times M_{0}$ defined as follows:

$$
M \ni q \longrightarrow\left(z, \exp \beta^{-1}(z) \cdot q\right) \in \mathcal{S} \times M_{0} .
$$

The case of $\mathscr{M}$ is similar.

Remark 4. In the case where $M$ is a Siegel domain of the second kind, the mapping $\Phi$ is an imbedding (Corollary 5, [8]) and the fibering $M \rightarrow \mathcal{S}$ corresponds to the realization of $M$ as a Siegel domain of the third kind constructed in [7].

\section{$\S 5$. The structure of the fiber $\mathscr{M}_{0}$.}

Let $H_{p}$ be as in $\S 3$. We can decompose $H_{p}$ as $H_{p}=H_{1}+H_{2}+H^{\prime}$, where $H_{1} \in \mathfrak{l}(p)_{c}, H_{2} \in \mathrm{t}_{-}$and $H^{\prime} \in \mathfrak{a}_{c} \cap \theta^{0}$. Since $\left[H_{p}, \mathfrak{l}(p)_{c}\right] \subset \mathfrak{l}(p)_{c}$, we have $\left[H_{2}, \mathfrak{l}(p)_{c}\right]=0$ and $a d H_{1} X=a d H_{p} X=a d E_{p} X$ for any $X \in \mathfrak{l}(p)_{c}$. Therefore $H_{1}=E_{p}$ and by (3.3), $\mathrm{H}_{2}=0$. Thus we can write

$$
H_{p}=E_{p}+H^{\prime}\left(H^{\prime} \in \mathfrak{a}_{c} \cap \theta^{0}\right) .
$$

Let $E^{\prime}$ (resp. $A$ ) be the real (resp. the imaginary) part of $H^{\prime}$. We can decompose $A$ as $A=v_{r}+I^{\prime}$, where $v_{r} \in \mathfrak{a}_{c} \cap \theta^{-1}(=\mathfrak{l})$ and $I^{\prime} \in \mathfrak{a}_{c} \cap \theta^{0}$. Then $H^{\prime}=E^{\prime}+\sqrt{-1}\left(v_{r}+I^{\prime}\right)$. 
Lemma 5.1. Both $v_{r}$ and $I^{\prime}$ are in a and $\left[v_{r}, I^{\prime}\right]=0$.

Proof. By direct calculations,

$$
\begin{aligned}
& {\left[E^{\prime}, A\right]=\left[H^{\prime}, A\right]=\left[H_{p}, A\right]=-v_{r},} \\
& {\left[E^{\prime}, v_{r}\right]=\left[H^{\prime}, v_{r}\right]-\sqrt{-1}\left[I^{\prime}, v_{r}\right]=-v_{r}-\sqrt{-1}\left[I^{\prime}, v_{r}\right] .}
\end{aligned}
$$

From the first equation, we have $v_{r} \in \mathfrak{g}(M)$ and hence $v_{r}, I^{\prime} \in \mathfrak{a}$. This combined with the second equation implies $\left[I^{\prime}, v_{r}\right] \subset g(M) \cap \sqrt{-1} g(M)=0$.

q.e.d.

By Lemma 5.1 and (5.1), we have

$$
\begin{aligned}
& A d\left(\exp \left(-\sqrt{-1} v_{r}\right)\right) H^{\prime}=E^{\prime}+\sqrt{-1} I^{\prime}, \\
& A d\left(\exp \left(-\sqrt{-1} v_{r}\right)\right) H_{p}=E^{\prime}+\sqrt{-1}\left(I^{\prime}+Z_{p}\right) .
\end{aligned}
$$

If we set

$$
\theta^{\prime 0}=A d\left(\exp \left(-\sqrt{-1} v_{r}\right)\right) \theta^{0}
$$

then we have from (5.2)

$$
\begin{aligned}
& \operatorname{ad}\left(E^{\prime}+\sqrt{-1} I^{\prime}\right)=-1 \quad \text { on } \mathfrak{u} \\
& \operatorname{ad}\left(E^{\prime}+\sqrt{-1} I^{\prime}\right)=0 \quad \text { on } \mathfrak{a}_{c} \cap \theta^{\prime 0}
\end{aligned}
$$

Since $I^{\prime}$ is contained in $\mathfrak{f}_{p}$ and since $\left[I^{\prime}, v_{r}\right]=0$, ad $I^{\prime}$ leaves $\mathfrak{u}$ and $\mathfrak{a}_{c} \cap \theta^{\prime 0}$ invariant and every eigenvalue of $a d I^{\prime}$ is purely imaginary. Hence if we set for $\lambda \in \boldsymbol{R}$,

then we have

$$
\begin{aligned}
U^{\lambda} & =\left\{X \in \mathfrak{u} ;\left[2 E^{\prime}, X\right]=\lambda X\right\} \\
V^{\lambda} & =\left\{X \in \mathfrak{a}_{c} \cap \theta^{\prime 0} ;\left[2 E^{\prime}, X\right]=\lambda X\right\},
\end{aligned}
$$

$$
\mathfrak{u}=\sum_{\lambda \in R} U^{\lambda} \quad \text { and } \quad \mathfrak{a}_{c} \cap \theta^{\prime 0}=\sum_{\lambda \in R} V^{\lambda}
$$

Lemma 5.2. (1) $\mathfrak{u}=U^{-2}+U^{-1}$ and $\mathfrak{a}_{c} \cap \theta^{\prime 0}=V^{-1}+V^{0}$.

(2) $a d\left(2 I^{\prime}\right)=\sqrt{-1}$ on $U^{-1}, a d\left(2 I^{\prime}\right)=-\sqrt{-1}$ on $V^{-1}$ and $\operatorname{ad}\left(2 I^{\prime}\right)=0$ on $U^{-2}+V^{0}$.

Proof. Let $v \in U^{\lambda}$. By (5.3), $a d\left(2 I^{\prime}\right) v=(\lambda+2) \sqrt{-1} v$. Hence

$$
\operatorname{ad}\left(2 I^{\prime}\right) \bar{v}=-(\lambda+2) \sqrt{-1} \bar{v} .
$$

We write $\bar{v}=v^{\prime}+X$, where $v^{\prime} \in u$ and $X \in a_{c} \cap \theta^{\prime 0}$. Clearly $v^{\prime} \in U^{\lambda}$ and $X \in V^{\lambda}$. Therefore by (5.3),

$$
\operatorname{ad}\left(2 I^{\prime}\right) v^{\prime}=(\lambda+2) \sqrt{-1} v^{\prime} \text { and } \operatorname{ad}\left(2 I^{\prime}\right) X=\lambda \sqrt{-1} X .
$$

It follows from (5.4) and (5.5), $(\lambda+2) v^{\prime}=0$ and $(\lambda+1) X=0$. Hence if $\lambda \neq-2,-1$, then $v=0$. If $\lambda=-1$, then $a d\left(2 I^{\prime}\right) v=\sqrt{-1} v$ and if $\lambda=-2$, then $a d\left(2 I^{\prime}\right) v=0$. Similarly, we have $V^{\lambda}=0$ if $\lambda \neq-1,0$ and $a d\left(2 I^{\prime}\right)=-\sqrt{-1}$ on $V^{-1}$ and $a d\left(2 I^{\prime}\right)=0$ on $V^{0}$. q.e.d.

As an immediate consequence of Lemma 5.2 , we have 
Lemma 5.3. (1) $\mathfrak{a}=\mathfrak{a}^{-2}+\mathfrak{a}^{-1}+\mathfrak{a}^{0}$, where $\mathfrak{a}^{\lambda}=\left\{X \in \mathfrak{a} ;\left[2 E^{\prime}, X\right]=\lambda X\right\}$.

(2) $\operatorname{ad}\left(2 I^{\prime}\right)=0$ on $\mathfrak{a}^{-2}+\mathfrak{a}^{0}$ and $\left(\operatorname{ad}\left(2 I^{\prime}\right)\right)^{2}=-1$ on $\mathfrak{a}^{-1}$.

(3) $\mathfrak{a}_{c} \cap \theta^{-1}=\mathfrak{a}_{c}^{-2}+\mathfrak{a}_{+}$and $\mathfrak{a}_{c} \cap \theta^{\prime 0}=\mathfrak{a}_{-}+\mathfrak{a}_{c}^{0}$, where

$$
\mathfrak{a}_{ \pm}=\left\{X \in \mathfrak{a}_{c}^{-1} ;\left[2 I^{\prime}, X\right]= \pm \sqrt{-1} X\right\} .
$$

It is clear that the subspace $t$ is invariant by ad a. We investigate the eigenvalues of ad $E^{\prime}$ on $t$.

Lemma 5.4. (1) ad $2 E^{\prime}=-1$ on $\mathrm{t}$.

(2) $\mathrm{t}_{ \pm}=\left\{X \in \mathrm{t}_{c} ;\left[2\left(Z_{p}+I^{\prime}\right), X\right]= \pm \sqrt{ }-1 X\right\}$.

Proof. Notice that $v_{r} \in \mathfrak{a}^{-2}$. Therefore by (2) of Lemma 4.1 and by (3) of Lemma 5.3 , we have $\left[\mathrm{t}, v_{r}\right]=0$. It follows that $\mathrm{t}_{+}$and $\mathrm{t}_{-}$are invariant by ad $X$ for any $X \in \mathfrak{a}_{c} \cap \theta^{\prime 0}$. Hence $\mathrm{t}_{+}$and $\mathrm{t}_{-}$are invariant by ad $E^{\prime}$ and by $\operatorname{ad}\left(I^{\prime}+Z_{p}\right)$. Since $I^{\prime}+Z_{p}$ is in $\mathfrak{f}_{p}$, all eigenvalues of ad $2\left(I^{\prime}+Z_{p}\right)$ are purely imaginary and both $t_{+}$and $t_{-}$are decomposed into the sum of eigenspaces of $\operatorname{ad} 2\left(I^{\prime}+Z_{p}\right)$. Let $v \in t_{+}$satisfying

$$
\operatorname{ad} 2\left(I^{\prime}+Z_{p}\right) v=\lambda \sqrt{ }-1 v \quad \text { for } \quad \lambda \in R \text {. }
$$

Then

$$
\text { ad } 2\left(I^{\prime}+Z_{p}\right) \bar{v}=-\lambda \sqrt{-1} \bar{v} .
$$

By (5.2), (5.6) and (5.7), we have

$$
\operatorname{ad}\left(2 E^{\prime}\right) v=(-2+\lambda) v \text { and } \operatorname{ad}\left(2 E^{\prime}\right) \bar{v}=-\lambda \bar{v},
$$

because $\bar{v} \in t_{\text {.. }}$ Thus we get $(\lambda-1) v=0$. Consequently, if $v \neq 0$, then $\lambda=1$ and $\operatorname{ad}\left(2 E^{\prime}\right) v=-v$. Considering $t_{-}=\overline{\mathrm{t}}_{+}$, we get (1) and (2). q.e.d.

By Lemmas 5.3 and 5.4, we have

$$
\begin{aligned}
& {\left[\mathfrak{a}^{-1}, \mathfrak{a}^{-1}\right] \subset \mathfrak{a}^{-2}, \quad[\mathrm{t}, \mathrm{t}] \subset \mathfrak{a}^{-2},} \\
& {\left[\mathfrak{a}^{-2}, \mathrm{t}\right]=\left[\mathfrak{a}^{-1}, \mathrm{t}\right]=\left[\mathfrak{a}^{-2}, \mathfrak{a}^{-1}\right]=0 .}
\end{aligned}
$$

Let $\mathscr{M}_{0}$ be the fiber defined by (4.4). By Remark $3, \mathscr{M}_{0}$ is connected and by Lemma 5.3, $\mathscr{M}_{0}$ is regarded as a domain of $\mathfrak{a}_{\mathfrak{c}}^{-2} \times\left(\mathfrak{a}_{+}+t_{+}\right)$.

Lemma 5.5. The domain $\mathcal{M}_{0}$ is invariant under the following transformations of $\mathrm{a}_{c}^{-2} \times\left(\mathrm{a}_{+}+\mathrm{t}_{+}\right)$.

(a) $(z, w) \rightarrow(z+a, w)$ for every $a \in \mathfrak{a}^{-2}$.

(b) $(z, w) \rightarrow\left(z+\frac{1}{2}[\bar{c}, c]+[\bar{c}, w], w+c\right)$ for every $c \in a_{+}+i_{+}$.

Proof. Let $X=c+\bar{c}$. By using (5.8), we get $\exp X=\exp c \cdot \exp \bar{c} \cdot \exp \frac{1}{2}[\bar{c}, c]$. We claim that $\bar{c}$ is contained in $\theta^{0}$. In fact, by Lemma 5.3, $a_{-} \subset \theta^{\prime 0}$. Since $\left[a_{-}, v_{r}\right]=0, a_{-} \subset A d\left(\exp \sqrt{-1} v_{r}\right) \theta^{\prime \prime}=\theta^{0}$. Therefore $\bar{c} \in a_{-}+t_{-} \subset \theta^{0}$. It follows 


$$
\begin{aligned}
\exp X \cdot h_{1}(z, w) & =\pi_{1}\left(\exp c \cdot \exp \frac{1}{2}[\bar{c}, c] \cdot \exp z \cdot \exp A d(\exp \bar{c}) w\right) \\
& =h_{1}\left(z+\frac{1}{2}[\bar{c}, c]+[\bar{c}, w], w+c\right) .
\end{aligned}
$$

Therefore $\mathscr{M}_{0}$ is invariant under the transformations of the form (b). For any $a \in \mathfrak{a}^{-2}$, the following equality holds clearly:

$$
\exp a \cdot h_{1}(z, w)=h_{1}(z+a, w) .
$$

We now set

$$
\begin{aligned}
& \Omega_{r}=\left\{y \in \mathfrak{a}^{-2} ;(\sqrt{-1} y, 0) \in \mathscr{M}_{0}\right\}+v_{r}, \\
& F_{r}\left(w, w^{\prime}\right)=\frac{\sqrt{-1}}{2}\left[w, \bar{w}^{\prime}\right] \quad \text { for } \quad w, w^{\prime} \in \mathfrak{a}_{+}+\mathfrak{t}_{+} .
\end{aligned}
$$

Then $\Omega_{r}$ is an open set of $\mathfrak{a}^{-2}$ containing $v_{r}$ and $F_{r}$ is an $\mathfrak{a}_{c}^{-2}$-valued hermitian form on $a_{+}+t_{+}$.

Definition. Let $\Omega$ be an open connected cone in a real vector space $V$ and let $F$ be a $V_{c}$-valued hermitian form on a complex vector space $W$ such that the condition " $F(w, w)=0$ " implies $w=0$. A pseudo-Siegel domain $D$ of the second kind associated with $\Omega$ and $F$ is a domain in $V_{c} \times W$ defined as follows:

$$
D=\left\{(z, w) \in V_{c} \times W ; \operatorname{Im} z-F(w, w) \in \Omega\right\} .
$$

A pseudo-Siegel domain $D$ of the second kind is a Siegel domain of the second kind if $\Omega$ is a convex cone containing no entire straight lines and $F$ is an $\Omega$-hermitian form, i.e., $F(w, w)$ is contained in the closure of $\Omega$.

Proposition 5.6. The fiber $\mathscr{M}_{0}$ is holomorphically equivalent to the pseudoSiegel domain of the second kind associated with $\Omega_{r}$ and $F_{r}$ defined by (5.9). In fact, $\mathcal{M}_{0}$ is represented as

$$
\mathscr{M}_{0}=\left\{(z, w) \in \mathfrak{a}_{c}^{-2} \times\left(\mathfrak{a}_{+}+t_{+}\right) ; \operatorname{Im} z-F_{r}(w, w)+v_{r} \in \Omega_{r}\right\} .
$$

Proof. Let $y \in \Omega_{r}$. Then $\left(\sqrt{-1}\left(y-v_{r}\right), 0\right) \in \mathscr{M}_{0}$. Using Lemma 5.3, we have

$$
\begin{aligned}
\exp & t E^{\prime} \cdot h_{1}\left(\sqrt{-1}\left(y-v_{r}\right), 0\right) \\
& =\pi_{1} \cdot \exp \left(A d\left(\exp \sqrt{-1} v_{r}\right)^{-1} t\left(H^{\prime}-\sqrt{-1} I^{\prime}\right)\right) \cdot \exp \sqrt{-1}\left(y-v_{r}\right) \\
& =\pi_{1} \cdot \exp \left(-\sqrt{-1} v_{r}\right) \cdot \exp t\left(H^{\prime}-\sqrt{-1} I^{\prime}\right) \cdot \exp \sqrt{-1} v_{r} \cdot \exp \sqrt{-1}\left(y-v_{r}\right) \\
& =\pi_{1} \cdot \exp \left(-\sqrt{-1} v_{r}\right) \cdot \exp A d\left(\exp t\left(H^{\prime}-\sqrt{-1} I^{\prime}\right)\right) \sqrt{-1} y \\
& =h_{1}\left(-\sqrt{-1} v_{r}+e^{-t} \sqrt{-1} y, 0\right),
\end{aligned}
$$

because $H^{\prime}-\sqrt{-1} I^{\prime}$ belongs to $\theta^{0}$. Therefore $\left(\sqrt{-1}\left(e^{-t} y-v_{r}\right), 0\right) \in \mathscr{M}_{0}$. This implies $e^{-t} y \in \Omega_{r}$ for any $t \in \boldsymbol{R}$, proving that $\Omega_{r}$ is a cone.

By a suitable transformation as in Lemma 5.5 , every point $(z, w)$ can be translated to $\left(\sqrt{-1}\left(\operatorname{Im} z-F_{r}(w, w)\right), 0\right)$. Therefore $(z, w) \in \mathscr{M}_{0}$ if and only if $\operatorname{Im} z-F_{r}(w, w)+v_{r} \in \Omega_{r}$. Now $\mathscr{M}_{0}$ is diffeomorphic to $\mathfrak{a}^{-2} \times \Omega_{r} \times\left(\mathfrak{a}_{+}+\mathfrak{t}_{+}\right)$under the 
following mapping :

$$
\mathscr{M}_{0} \ni(z, w) \longrightarrow\left(\operatorname{Re} z, \operatorname{Im} z-F_{r}(w, w)+v_{r}, w\right) \in \mathfrak{a}^{-2} \times \Omega_{r} \times\left(\mathfrak{a}_{+}+\mathfrak{t}_{+}\right) .
$$

Therefore $\Omega_{r}$ is connected.

Assume that $F_{r}(w, w)=0$. Let $X=w+\bar{w}$ and $Y=\sqrt{-1} w-\sqrt{-1} \bar{w}$. We define a mapping $\phi$ of $C$ into $M$ by

$$
\phi(z)=\exp x X \cdot \exp y Y(p) \quad \text { for } \quad z=x+\sqrt{-1} y .
$$

Since $[w, \bar{w}]=0$, we get from the proof of Lemma 5.5 ,

$$
h_{1}^{-1} \circ \Phi \circ \psi(z)=(0, x w+\sqrt{-1} y w)=(0, z w) .
$$

Since $h_{1}^{-1} \diamond \Phi$ is an immersion, $\psi(z)$ must be holomorphic. This means that $\phi(z)=p$ for any $z \in C$ and hence $w=0$, completing the proof.

q.e.d.

\section{$\S 6$. The symmetric Siegel domain $S$ isomorphic to $M(p)$ and the structure of $t$.}

It is well known that the hermitian symmetric space $M(p)$ of the non-compact type is holomorphically isomorphic to a symmetric Siegel domain $S$ of the second kind. Therefore by Kaup-Matsushima-Ochiai [2], there exists $E_{s}$ of $\mathfrak{I}(p)$ such that

$$
\begin{aligned}
& \mathfrak{I}(p)=2^{-2}+g^{-1}+z^{0}+z^{1}+2^{2}, \\
& \mathfrak{s}^{\lambda}=\left\{X \in \mathfrak{l}(p) ;\left[E_{s}, X\right]=\lambda X\right\} .
\end{aligned}
$$

Note that $\operatorname{dim}_{R} \underline{2}^{-2}=\operatorname{dim}_{R} \underline{2}^{2}$ and $\operatorname{dim}_{R^{2}}{ }^{-1}=\operatorname{dim}_{R^{2}}$, because $\mathfrak{I}(p)$ is semi-simple. Moreover there exists $I_{s}$ of $z^{0}$ such that

$$
\begin{aligned}
& \text { ad } I_{s}=0 \text { on } \mathfrak{g}^{-2}+\mathfrak{s}^{0}+\mathfrak{s}^{2} \\
& \left(\text { ad } I_{s}\right)^{2}=-1 \text { on } \mathfrak{g}^{-1}+\mathfrak{s}^{1} .
\end{aligned}
$$

Define linear transformations $P$ and $\bar{P}$ of $\gtrless_{c}^{-1}+\gtrless_{c}^{1}$ by

$$
\begin{aligned}
& P(X)=\frac{1}{2}\left(X-\sqrt{-1}\left[I_{s}, X\right]\right) \\
& \bar{P}(X)=\frac{1}{2}\left(X+\sqrt{-1}\left[I_{s}, X\right]\right),
\end{aligned}
$$

and set

$$
\begin{aligned}
& \theta_{s}^{-1}=\mathfrak{a}_{c}^{-2}+P\left(\mathfrak{g}^{-1}\right) \\
& \theta_{s}^{0}=\bar{P}\left(\mathfrak{g}^{-1}\right)+\mathfrak{s}_{c}^{0}+P\left(\mathfrak{g}^{1}\right) \\
& \theta_{s}^{1}=\bar{P}\left(\mathfrak{g}^{1}\right)+\mathfrak{a}_{c}^{2} \\
& H_{s}=\frac{1}{2}\left(E_{s}+\sqrt{-1} I_{s}\right) .
\end{aligned}
$$

By (6.1) and (6.2), we have 


$$
\begin{aligned}
& \mathfrak{l}(p)_{c}=\theta_{s}^{-1}+\theta_{s}^{0}+\theta_{s}^{1} \\
& \text { ad } H_{s} X=\lambda X \quad \text { for } X \in \theta_{s}^{\lambda} .
\end{aligned}
$$

The Siegel domain $S$ is regarded as a domain in $a_{c}^{-2}+P\left(a^{-1}\right)$ defined by

$$
S=\left\{z+w \in \mathfrak{B}_{c}^{-2}+P\left(\mathfrak{g}^{-1}\right) ; \operatorname{Im} z-F_{s}(w, w) \in \Omega_{s}\right\},
$$

where $\Omega_{s}$ is an open convex cone in $\mathfrak{g}^{-2}$ containing no entire straight lines and $F_{s}$ is an $\Omega_{s}$-hermitian form on $P\left(g^{-1}\right)$ given by $F_{s}\left(w, w^{\prime}\right)=\frac{\sqrt{-1}}{2}\left[w, \bar{w}^{\prime}\right]$ (Tanaka [12]). By Lemma 2.1 of [7], for every $v$ of $\Omega_{s}$, there exists a unique $\hat{v} \in \mathfrak{g}^{2}$ such that

$$
[\hat{v}, v]=E_{s} .
$$

We then have (see, $\S 2$ of [7])

$$
\mathfrak{s}^{-2}=\left[v,\left[v, \mathfrak{z}^{2}\right]\right] \text { and } \mathfrak{s}^{2}=\left[\hat{v},\left[\hat{v}, \mathfrak{s}^{-2}\right]\right] \quad \text { for } v \in \Omega_{s} .
$$

We may assume that the point $p$ of $M(p)$ corresponds to $\sqrt{-1} v_{s}$ of $S$ where $v_{s} \in \Omega_{s}$. Then by Proposition 2.4 of [7],

$$
Z_{p}=\frac{1}{2}\left(I_{s}+v_{s}-\hat{v}_{s}\right) .
$$

We put

$$
\delta_{s}=\exp \sqrt{-1} v_{s} \cdot \exp \frac{\sqrt{-1}}{2} \hat{v}_{s} .
$$

Using (6.1), (6.2) and (6.4), we get

$$
\text { Ad } \delta_{s}^{-1} Z_{p}=\frac{1}{2}\left(I_{s}-\sqrt{-1} E_{s}\right)=-\sqrt{-1} H_{s} .
$$

Therefore by (6.3) and (6.7), we have

$$
\operatorname{Ad} \delta_{s} \theta_{s}^{-1}=\mathfrak{l}_{+}, \quad \text { Ad } \delta_{s} \theta_{s}^{0}=\mathfrak{l}(p)_{c}, \quad \text { Ad } \delta_{s} \theta_{s}^{1}=\mathfrak{l}_{-} .
$$

Let $B^{s}$ be the closed subgroup of $L_{c}$ defined by

$$
B^{s}=\left\{a \in L_{c} ; a\left(\theta_{s}^{0}+\theta_{s}^{1}\right)=\theta_{s}^{0}+\theta_{s}^{1}\right\} .
$$

It is easy to see that $B^{s}$ is connected and

$$
\text { Ad } \delta_{s} B^{s}=K_{c} \cdot L_{-} .
$$

Define a holomorphic mapping $h_{s}$ of $\theta_{s}^{-1}$ to $L_{c} / B^{s}$ by

$$
h_{s}(z)=\pi_{s} \cdot \exp z \quad \text { for } \quad z \in \theta_{s}^{-1},
$$

where $\pi_{s}$ denotes the projection: $L_{c} \rightarrow L_{c} / B^{s}$. The mapping $h_{s}$ is an imbedding of $\theta_{s}^{-1}$ onto an open dense subset of $L_{c} / B^{s}$ and under the natural action of $L$ on $S$, the restriction of $h_{s}$ to $S$ is $L$-equivariant (Tanaka [12]). Let $\tilde{\delta}_{s}$ be a holomorphic diffeomorphism of $L_{c} / B^{s}$ onto $L_{c} / K_{c} \cdot L$ - given by 


$$
L_{c} / B^{s} \ni g B^{s} \stackrel{\tilde{\delta}_{s}}{\longrightarrow} g \delta_{s}^{-1} K_{c} \cdot L_{-} \in L_{c} / K_{c} \cdot L_{-} .
$$

The mapping $\tilde{o}_{s}$ is $L_{c}$-equivariant. It should be noted that $L_{c} \cap B=K_{c} \cdot L_{-}$. We then have the following commutative diagram:

$$
\begin{aligned}
& L_{c} / B^{s} \stackrel{\tilde{\delta}_{s}}{\longrightarrow} L_{c} / K_{c} \cdot L_{-} \sqsubset G_{c} / B
\end{aligned}
$$

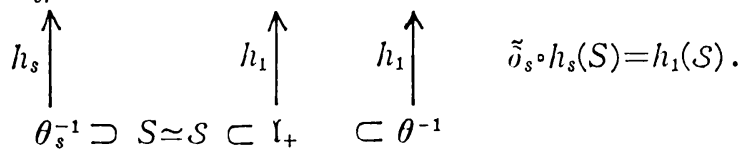

We now consider the decomposition of $t(=[\mathfrak{r}, \mathfrak{l}(p)])$ into the sum of eigenspaces of ad $E_{s}$.

Lemma 6.1. (1) $\mathrm{t}=\mathrm{t}^{-1}+\mathrm{t}^{0}+\mathrm{t}^{1}$, where $\mathrm{t}^{2}=\left\{X \in \mathrm{t} ;\left[E_{s}, X\right]=\lambda X\right\}$.

(2) $\mathrm{t}^{-1}=\left[\mathrm{t}^{1}, v_{s}\right]$ and $\mathrm{t}^{1}=\left[\mathrm{t}^{-1}, \hat{v}_{s}\right]$.

(3) $\left[\mathrm{t}^{0}, \mathrm{t}^{-1}+\mathrm{t}^{1}\right]=0$ and $\left[\mathrm{t}^{0}, \mathfrak{g}^{-2}+\left[\mathfrak{g}^{-2}, \mathfrak{g}^{2}\right]+\mathfrak{g}^{2}\right]=0$.

Proof. Since $E_{s}$ is a real diagonal element of the semi-simple Lie algebra $\mathfrak{I}(p)$, we have by Lemma 1.5 of [6],

$$
\mathrm{t}=\sum_{\lambda \in R} \mathrm{t}^{\lambda}, \quad \mathrm{t}^{i}=\left\{X \in \mathrm{t} ;\left[E_{s}, X\right]=\lambda X\right\} .
$$

Let $w \in t^{\lambda}$. Then by Lemma 5.4, we have $w=w_{+}+w_{-}$, where

$$
w_{ \pm}=\frac{1}{2}\left(u \mp 2 \sqrt{-1}\left[I^{\prime}+Z_{p}, w\right]\right) .
$$

For any $X \in \mathbb{2}^{2}$,

$$
\text { Ad } \delta_{s} X=X+\sqrt{-1}\left[v_{s}, X\right]-\frac{1}{2}\left[v_{s},\left[v_{s}, X\right]\right]
$$

By using (6.6),

$$
2 w_{-}=w+2 \sqrt{-1}\left[I^{\prime}, w\right]+\sqrt{-1}\left[I_{s}, w\right]+\sqrt{-1}\left[v_{s}, w\right]-\sqrt{-1}\left[\hat{v}_{s}, w\right] .
$$

Since $\left[A d \delta_{s} X, w_{-}\right]=0$ by (4.3) and (6.8), we get by considering the $t^{\lambda \pm 4}$-component of $\left[A d \delta_{s} X, w_{-}\right],\left[X,\left[\hat{v}_{s}, w\right]\right]=0$ and $\left[\left[v_{s},\left[v_{s}, X\right]\right],\left[v_{s}, w\right]\right]=0$. These show that $\left[\left[\hat{v}_{s}, w\right], \Sigma^{2}\right]=0$ and $\left[\left[v_{s}, w\right], g^{-2}\right]=0$, because of (6.5). Therefore we have proved $\left[\left[\mathrm{t}, \hat{v}_{s}\right], \mathfrak{s}^{2}\right]=0$ and $\left[\left[\mathrm{t}, v_{s}\right], \mathfrak{s}^{-2}\right]=0$. From (6.5), we also know that $\mathfrak{s}^{-2}=\left[v_{s}, \mathfrak{s}^{0}\right]$ and $\mathfrak{s}^{2}=\left[\hat{v}_{s}, \mathfrak{e}^{0}\right]$. It follows that

$$
\begin{aligned}
{\left[\left[1, s^{2}\right], s^{2}\right] } & =\left[\left[1, s^{2}\right],\left[\hat{v}_{s}, s^{0}\right]\right] \\
& \subset\left[\left[\left[1, s^{2}\right], \mathfrak{s}^{0}\right], \hat{v}_{s}\right] \subset\left[\left[t, \mathfrak{s}^{2}\right], \hat{v}_{s}\right]=0 .
\end{aligned}
$$

Similarly we get $\left[\left[1, \mathfrak{g}^{-2}\right], \mathfrak{g}^{-2}\right]=0$. Let $\lambda_{1}$ (resp. $\lambda_{2}$ ) be the maximal (resp. the minimal) $\lambda$ such that $\mathrm{t}^{\lambda} \neq 0$. Then $\left[\mathrm{t}^{\lambda_{1}}, \mathfrak{g}^{2}\right]=0$. Therefore if we set $\mathfrak{z}=\mathfrak{g}^{-2}+\left[\mathfrak{g}^{-2}, \mathfrak{g}^{2}\right]+\mathfrak{g}^{2}$, then $\mathfrak{t}^{\lambda_{1}}+\left[f^{\lambda_{1}}, \mathfrak{g}^{-2}\right]$ is an ad $\mathfrak{z}_{\mathrm{j}}$-invariant subspace. Since $E_{s} \in\left[\mathfrak{z}^{-2}, \Omega^{2}\right]$, the trace of ad $E_{s}$ on $t^{\lambda_{1}}+\left[t^{\lambda_{1}}, g^{-2}\right]$ is equal to zero. Therefore 


$$
\lambda_{1}\left(\operatorname{dim}_{R} t^{\lambda_{1}}+\operatorname{dim}_{R}\left[\dagger^{\lambda_{1}}, g^{-2}\right]\right)=2 \operatorname{dim}_{R}\left[1^{\lambda_{1}}, g^{-2}\right] .
$$

It follows that $\lambda_{1}<2$. Similarly we have $\lambda_{2}>-2$. Consequently, $\left[\mathrm{t}^{\lambda_{1}-2}, g^{-2}\right]=0$ because $\lambda_{1}-4<-2$. Then $\mathrm{t}^{\lambda_{1}}+\mathrm{t}^{\lambda_{1}-2}$ and $\left[\mathrm{t}^{\lambda_{1}-2}, \mathrm{~g}^{2}\right]+\mathrm{t}^{\lambda_{1}-2}$ are ad $z$-invariant. Hence we have

$$
\begin{aligned}
& \lambda_{1}\left(\operatorname{dim}_{R} \mathrm{t}^{\lambda_{1}}+\operatorname{dim}_{R} \mathrm{t}^{\lambda_{1}-2}\right)=2 \operatorname{dim}_{R} \mathrm{t}^{\lambda_{1}-2} . \\
& \lambda_{1}\left(\operatorname{dim}_{R}\left[\mathrm{t}^{\lambda_{1}-2}, \mathcal{S}^{2}\right]+\operatorname{dim}_{R} \mathrm{t}^{\lambda_{1}-2}\right)=2 \operatorname{dim}_{R} \mathrm{t}^{\lambda_{1}-2} .
\end{aligned}
$$

From (6.11) and (6.12), we get $t^{\lambda_{1}-2}=\left[t^{\lambda_{1}}, g^{-2}\right]$ and if $\lambda_{1} \neq 0$, then $t^{\lambda_{1}}=\left[t^{\lambda_{1}-2}, \Xi^{2}\right]$. Assume $\lambda_{1} \neq 0$. Then using (6.5), we have

$$
\begin{aligned}
\mathrm{t}^{\lambda_{1}-2}=\left[\mathrm{f}^{\lambda_{1}},\left[v_{s},\left[v_{s}, \mathfrak{B}^{2}\right]\right]\right] & \subset\left[\left[\mathrm{t}^{\lambda_{1}}, v_{s}\right],\left[v_{s}, \mathfrak{P}^{2}\right]\right]+\left[v_{s}, \mathrm{t}^{\lambda_{1}}\right] \\
& \subset\left[\left[\left[\mathrm{t}^{\lambda_{1}}, v_{s}\right], \mathfrak{P}^{2}\right], v_{s}\right]+\left[v_{s}, \mathrm{t}^{\lambda_{1}}\right] \\
& =\left[v_{s}, \mathrm{t}^{\lambda_{1}}\right] .
\end{aligned}
$$

Therefore $\mathrm{t}^{\lambda_{1}-2}=\left[v_{s}, \mathrm{t}^{\lambda_{1}}\right]$. Similarly, $\mathrm{t}^{\lambda_{1}}=\left[\mathrm{t}^{\lambda_{1}-2}, \hat{v}_{s}\right]$. It follows that $\operatorname{dim}_{R} \mathrm{t}^{\lambda_{1}}=$ $\operatorname{dim}_{R} t^{\lambda_{1}-2}$ and hence $\lambda_{1}=1$. By the same way, we can show $\lambda_{2}=-1$ or 0 . We have proved that if $\mathrm{t}^{\lambda} \neq 0$, then $-1 \leqq \lambda \leqq 1$ and $\mathrm{t}^{1}=\left[\hat{v}_{s}, \mathrm{t}^{-1}\right]$ and $\mathrm{t}^{-1}=\left[v_{s}, \mathrm{t}^{1}\right]$. Consequently, if $-1<\lambda<1$, then $\left[t^{2}, z^{2}+z^{-2}\right]=0$ and hence $\left[t^{\lambda}, \xi\right]=0$. This means $\lambda=0$.

It remains to show that $\left[\mathrm{t}^{0}, \mathrm{t}^{1}+\mathrm{t}^{-1}\right]=0$. Recall that $[\mathrm{t}, \mathrm{t}] \subset \mathfrak{a}$ by (1) of Lemma 4.1. Since ad $E_{s}= \pm 1$ on $\left[\mathrm{t}^{0}, \mathrm{t}^{ \pm 1}\right]$, this means $\left[\mathrm{t}^{0}, \mathrm{t}^{-1}+\mathrm{t}^{1}\right]=0$. q.e. $\mathrm{d}$

Next we shall prove the following

Lemma 6.2. Let $t_{+}$and $t_{-}$be as in $\S 4$. Then

$$
\begin{aligned}
& \mathrm{t}_{+}=A d \delta_{s} \mathrm{t}_{c}^{-1}+\mathrm{t}_{+} \cap \mathrm{t}_{c}^{0} \\
& \mathrm{t}_{-}=A d \delta_{s} \mathrm{t}_{c}^{1}+\mathrm{t}_{-} \cap \mathrm{t}_{c}^{0} .
\end{aligned}
$$

Proof. Every element of $\mathfrak{g}^{0}$ leaves $t^{\lambda}$ invariant because $\left[E_{s}, \mathfrak{g}^{0}\right]=0$. In particular, $\left[I_{s}, \mathrm{t}^{\lambda}\right] \subset \mathrm{t}^{\lambda}$. Since $I_{s}$ is contained in the isotropy subalgebra at $\sqrt{-1} v_{s}$, we can decompose $t_{c}^{-1}$ and $t_{c}^{1}$ as follows:

$$
\begin{aligned}
& \mathrm{t}_{c}^{-1}=\sum_{\lambda \in R} T_{\lambda}^{-1}, \quad T_{\lambda}^{-1}=\left\{X \in \mathrm{t}_{c}^{-1} ;\left[I_{s}, X\right]=\lambda \sqrt{-1} X\right\}, \\
& \mathrm{t}_{c}^{1}=\sum_{i \in R} T_{\lambda}^{1}, \quad T_{\lambda}^{1}=\left\{X \in \mathrm{t}_{c}^{1} ;\left[I_{s}, X\right]=\lambda \sqrt{-1} X\right\} .
\end{aligned}
$$

Note that $\overline{T_{i}^{-1}}=T_{-\lambda}^{-1}$ and $\overline{T_{\lambda}^{1}}=T_{-\lambda}^{1}$. From (6.7), we have

$$
E_{p}=\sqrt{-1} Z_{p}=\frac{1}{2} A d \delta_{s}\left(E_{s}+\sqrt{-1} I_{s}\right) .
$$

Let $u \in T_{i}^{-1}$. Then

$$
\left[E_{p}, A d \delta_{s} u\right]=-\frac{1}{2}(\lambda+1) A d \delta_{s} u
$$

and 


$$
\left[E_{p}, A d \delta_{s} \bar{u}\right]=\frac{1}{2}(\lambda-1) A d \delta_{s} \bar{u} .
$$

We know from (3.2) and (3.3) that if $u \neq 0$, then $-1<\frac{1}{2}(\lambda-1),-\frac{1}{2}(\lambda+1)<1$. This implies $-1<\lambda<1$ and hence $-1<\frac{1}{2}(\lambda-1),-\frac{1}{2}(\lambda+1)<0$. It follows from (3.3) that $u$ and $\bar{u}$ are contained in $t_{+}$. Therefore $A d \delta_{s} t_{c}^{-1} \subset t_{+}$. We can show $A d \delta_{s} t_{c}^{1} \subset t_{-}$similarly. Let $w \in \mathrm{t}_{c}^{0}$. Then $w=w_{+}+w$ - where

$$
w_{ \pm}=\frac{1}{2}\left(w \mp 2 \sqrt{-1}\left[Z_{p}+I^{\prime}, w\right]\right) .
$$

By Lemma 6.1, $w=A d \delta_{s} w$. Therefore

$$
\left[Z_{p}, w\right]=\frac{1}{2} \text { Ad } \delta_{s}\left[I_{s}-\sqrt{-1} E_{s}, w\right]=\frac{1}{2}\left[I_{s}, w\right] \in \mathrm{t}_{c}^{0} .
$$

This shows that $w_{ \pm} \in \mathrm{t}_{c}^{0}$. Hence we get $\mathrm{t}_{c}^{0}=\mathrm{t}_{+} \cap \mathrm{t}_{c}^{0}+\mathrm{t}_{-} \cap \mathrm{t}_{c}^{0}$.

q.e.d.

\section{$\S 7$. Relization of $\mathscr{M}$ as a pseudo-Siegel domain.}

We put

$$
V=\mathfrak{3}^{-2}+\mathfrak{t}^{-1}+\mathfrak{a}^{-2}
$$

and define an open set $\Omega$ of $V$ by

$$
\Omega=\left\{a+b+c ; a \in \Omega_{s}, b \in \mathrm{t}^{-1}, c \in \mathfrak{a}^{-2}, c-\frac{1}{2}[[b, \hat{a}], b] \in \Omega_{r}\right\},
$$

where $\hat{a} \in \mathfrak{g}^{2}$ given by (6.4). Making use of the uniqueness, we know that $\widehat{t a}=\frac{1}{t} \hat{a}$ for any $t>0$. Hence $\Omega$ is a cone. Next we put

$$
W=P\left(\mathfrak{g}^{-1}\right)+\mathrm{t}_{c}^{0} \cap \mathrm{t}_{+}+\mathfrak{a}_{+} .
$$

Since $\left[\mathrm{t}^{0}, \mathfrak{g}^{-1}\right] \subset \mathrm{t}^{-1}$, we have $[W, \bar{W}] \subset V_{c}$. Thus we can define a $V_{c}$-valued hermitian form $F$ on $W$ by

$$
F\left(w, w^{\prime}\right)=\frac{\sqrt{-1}}{2}\left[w, \bar{w}^{\prime}\right] \quad \text { for } \quad w, w^{\prime} \in W .
$$

By using $Q$ and $F$, define an open set $D$ of $V_{c} \times W$ by

$$
D=\left\{(z, w) \in V_{c} \times W ; \operatorname{Im} z-F(w, w) \in \Omega\right\} .
$$

Let $\delta=\exp \sqrt{-1} v_{r} \cdot \exp \sqrt{-1} v_{s} \cdot \exp \frac{\sqrt{-1}}{2} \hat{v}_{s}$, where $v_{r}$ and $v_{s}$ are the elements of $\Omega_{r}$ and $\Omega_{s}$ as in $\S 5$ and $\S 6$ respectively. It is easy to see that

$$
\text { Ad } \delta\left(V_{c}+W\right)=\theta^{-1} \text {. }
$$

Therefore $V_{c}+W$ is abelian. Let us put

$$
B_{0}=A d \delta^{-1} B
$$

and define a holomorphic mapping $h_{0}$ of $V_{c}+W$ to $G_{c} / B_{0}$ by 


$$
h_{0}(z)=\pi_{0} \cdot \exp z
$$

where $\pi_{0}$ denotes the projection: $G_{c} \rightarrow G_{c} / B_{0}$. Then $h_{0}$ is an imbedding. It is clear that $B^{s}=L_{c} \cap B_{0}$ and the restriction of $h_{0}$ to $s_{c}^{-2}+P\left(g^{-1}\right)$ coincides with $h_{s}$. We shall examine the action of $A(S)$ on $h_{0}(D)$, where $A(S)$ means the connected subgroup of $L$ corresponding to $\mathfrak{g}^{-2}+\mathfrak{Z}^{-1}+\mathfrak{g}^{0}$. In what follows, we represent a vector of $V_{c}+W$ by a system of vectors $\left(z_{1}, z_{2}, z_{3}, w_{1}, w_{2}, w_{3}\right)$ under the identification :

$$
V_{c}+W \cong \mathfrak{g}_{c}^{-2} \times \mathrm{t}_{c}^{-1} \times \mathfrak{a}_{c}^{-2} \times P\left(\mathfrak{g}^{-1}\right) \times\left(\mathrm{t}_{c}^{0} \cap \mathrm{t}_{+}\right) \times \mathfrak{a}_{+} .
$$

Lemma 7.1. Let $f_{-2}=\exp c_{-2}, \quad f_{-1}=\exp c_{-1}$ and $f_{0}=\exp c_{0}$, where $c_{-2} \in z^{-2}$, $c_{-1} \in \mathfrak{Z}^{-1}$ and $c_{0} \in \mathfrak{Z}^{0}$. Then $f_{j}$ leaves $h_{0}\left(V_{c}+W\right)$ invariant and induces an affine transformation $\tilde{f}_{j}$ of $V_{c}+W$ as follows $(j=-2,-1,0)$ :

$$
\begin{aligned}
\tilde{f}_{-2}\left(z_{1}, z_{2}, z_{3}, w_{1}, w_{2}, w_{3}\right)=\left(z_{1}+c_{-2}, z_{2}, z_{3}, w_{1}, w_{2}, w_{3}\right), \\
\tilde{f}_{-1}\left(z_{1}, z_{2}, z_{3}, w_{1}, w_{2}, w_{3}\right)=\left(z_{1}^{\prime}, z_{2}^{\prime}, z_{3}^{\prime}, w_{1}^{\prime}, w_{2}^{\prime}, w_{3}^{\prime}\right) \\
z_{1}^{\prime}=z_{1}+\frac{1}{2}\left[\bar{P}\left(c_{-1}\right), P\left(c_{-1}\right)\right]+\left[\bar{P}\left(c_{-1}\right), w_{1}\right] \\
z_{2}^{\prime}=z_{2}+\left[\bar{P}\left(c_{-1}\right), w_{2}\right] \\
z_{3}^{\prime}=z_{3} \\
w_{1}^{\prime}=w_{1}+P\left(c_{-1}\right) \\
w_{2}^{\prime}=w_{2} \\
w_{3}^{\prime}=w_{3}, \\
\tilde{f}_{0}\left(z_{1}, z_{2}, z_{3}, w_{1}, w_{2}, w_{3}\right)=\left(\text { Ad } f_{0} z_{1}, \text { Ad } f_{0} z_{2}, z_{3}, \text { Ad } f_{0} w_{1}, w_{2}, w_{3}\right) .
\end{aligned}
$$

Proof. The assertions for $f_{-2}$ and $f_{0}$ are obvious. We shall prove for the case of $f_{-1}$. Since $c_{-1}=P\left(c_{-1}\right)+\bar{P}\left(c_{-1}\right)$ and since $\left[\mathfrak{g}^{-1},\left[\mathfrak{g}^{-1}, \mathfrak{g}^{-1}\right]\right]=0$, $f_{-1}=\exp P\left(c_{-1}\right) \cdot \exp \bar{P}\left(c_{-1}\right) \cdot \exp \frac{1}{2}\left[\bar{P}\left(c_{-1}\right), P\left(c_{-1}\right)\right]$. Therefore

$$
\begin{aligned}
f_{-1} \cdot h_{0}\left(z_{1}, z_{2}, z_{3}, w_{1}, w_{2}, w_{3}\right) \\
=\pi_{0} \cdot \exp \left(z_{1}+\frac{1}{2}\left[\bar{P}\left(c_{-1}\right), P\left(c_{-1}\right)\right]\right) \cdot \exp P\left(c_{-1}\right) \cdot \exp z_{2} \cdot \exp z_{3} \cdot \exp w_{3} \\
\quad \times \exp \bar{P}\left(c_{-1}\right) \cdot \exp w_{1} \cdot \exp w_{2} .
\end{aligned}
$$

Here we used $\left[\bar{P}\left(c_{-1}\right), z_{2}\right] \in\left[g_{c}^{-1}, \mathrm{t}_{c}^{-1}\right] \subset \mathrm{t}_{c}^{-2}=0$. Since $\exp \bar{P}\left(c_{-1}\right) \in B_{0}$ and since $\left[\bar{P}\left(c_{-1}\right),\left[\bar{P}\left(c_{-1}\right), w_{2}\right]\right] \in \mathrm{t}_{c}^{-2}=0$, we have

$$
\begin{aligned}
\exp & \bar{P}\left(c_{-1}\right) \cdot \exp w_{1} \cdot \exp w_{2} \\
& \equiv \exp \left(w_{1}+\left[\bar{P}\left(c_{-1}\right), w_{1}\right]\right) \cdot \exp \left(w_{2}+\left[\bar{P}\left(c_{-1}\right), w_{2}\right]\right) \\
& \equiv \exp w_{1} \cdot \exp \left[\bar{P}\left(c_{-1}\right), w_{1}\right] \cdot \exp w_{2} \cdot \exp \left[\bar{P}\left(c_{-1}\right), w_{2}\right] \quad\left(\bmod B_{0}\right)
\end{aligned}
$$

It follows 


$$
\begin{aligned}
f_{-1} \cdot h_{0}\left(z_{1}, z_{2}, z_{3}, w_{1}, w_{2}, w_{3}\right) \\
\equiv \exp \left(z_{1}+\frac{1}{2}\left[\bar{P}\left(c_{-1}\right), P\left(c_{-1}\right)\right]+\left[\bar{P}\left(c_{-1}\right), w_{1}\right]\right) \cdot \exp \left(w_{1}+P\left(c_{-1}\right)\right) \\
\quad \times \exp \left(z_{2}+\left[\bar{P}\left(c_{-1}\right), w_{2}\right]\right) \cdot \exp w_{2} \cdot \exp z_{3} \cdot \exp w_{3} \quad\left(\bmod B_{0}\right) .
\end{aligned}
$$

q.e.d.

Next we verify

Lemma 7.2. Let $f_{-2}, f_{-1}$ and $f_{0}$ be as in Lemma 7.1. Then $\tilde{f}_{j}(D)=D$ $(j=-2,-1,0)$.

Proof. (a) The case of $f_{-2}$. Clear.

(b) The case of $f_{-1}$. Let $z=z_{1}+z_{2}+z_{3}$ and let $w=w_{1}+w_{2}+w_{3}$. Then $F(w, w)=F\left(w_{1}, w_{1}\right)+F\left(w_{2}, w_{2}\right)+F\left(w_{3}, w_{3}\right)+F\left(w_{1}, w_{2}\right)+F\left(w_{2}, w_{1}\right)$. By Lemma 7.1, $F\left(w_{1}^{\prime}, w_{2}^{\prime}\right)+F\left(w_{2}^{\prime}, w_{1}^{\prime}\right)=F\left(w_{1}, w_{2}\right)+F\left(w_{2}, w_{1}\right)+F\left(P\left(c_{-1}\right), w_{2}\right)+F\left(w_{2}, P\left(c_{-1}\right)\right)$ and $\operatorname{Im} z_{2}^{\prime}=\operatorname{Im} z_{2}+\operatorname{Im}\left[\bar{P}\left(c_{-1}\right), w_{2}\right]=\operatorname{Im} z_{2}+F\left(P\left(c_{-1}\right), w_{2}\right)+F\left(w_{2}, P\left(c_{-1}\right)\right)$. It is clear that $\operatorname{Im} z_{1}^{\prime}-F\left(w_{1}^{\prime}, w_{1}^{\prime}\right)=\operatorname{Im} z_{1}-F\left(w_{1}, w_{1}\right)$. Since $z_{3}^{\prime}=z_{3}, w_{2}^{\prime}=w_{2}$ and $w_{3}^{\prime}=w_{3}$, combining the above equalities we get $\operatorname{Im} z^{\prime}-F\left(w^{\prime}, w^{\prime}\right)=\operatorname{Im} z-F(w, w)$, where $\left(z^{\prime}, w^{\prime}\right)=$ $\tilde{f}_{-1}(z, w)$.

(c) The case of $f_{0}$. Let $\left(z^{\prime}, w^{\prime}\right)=\tilde{f}_{0}(z, w)$. It is clear that $\operatorname{Im} z^{\prime}-F\left(w^{\prime}, w^{\prime}\right)$ $=A d f_{0}(\operatorname{Im} z-F(w, w))$. Therefore it is sufficient to show that $A d f_{0} \Omega=\Omega$. Let $v=v_{1}+v_{2}+v_{3}$, where $v_{1} \in \mathfrak{g}^{-2}, v_{2} \in \mathrm{t}^{-1}$ and $v_{3} \in \mathfrak{a}^{-2}$. We set $A d f_{0} v_{i}=v_{i}^{\prime}$. Since $f_{0}$ is an automorphism of $S, v_{1}^{\prime}$ belongs to $\Omega_{s}$ if $v_{1}$ belongs to $\Omega_{s}$ and by (6.4), $\hat{v}_{1}^{\prime}=A d f_{0} \hat{v}_{1}$. Therefore if $v_{1} \in \Omega_{s}$, then

$$
\begin{aligned}
v_{3}^{\prime}-\frac{1}{2}\left[\left[v_{2}^{\prime}, \hat{v}_{1}^{\prime}\right], v_{2}^{\prime}\right] & =v_{3}-\frac{1}{2}\left[\left[\operatorname{Ad} f_{0} v_{2}, \operatorname{Ad} f_{0} \hat{v}_{1}\right], \operatorname{Ad} f_{0} v_{2}\right] \\
& =v_{3}-\frac{1}{2}\left[\left[v_{2}, \hat{v}_{1}\right], v_{2}\right] .
\end{aligned}
$$

This means $A d f_{0} v \in \Omega$ if $v \in \Omega$.

q.e.d.

We set

$$
D_{0}=\left\{\left(z_{1}, z_{2}, z_{3}, w_{1}, w_{2}, w_{2}\right) \in D ; z_{1}=\sqrt{-1} v_{s} \text { and } w_{1}=0\right\} .
$$

Let $\eta_{0}$ denote the projection: $\left(z_{1}, z_{2}, z_{3}, w_{1}, w_{2}, w_{3}\right) \rightarrow\left(z_{1}, w_{1}\right)$. Then $\eta_{0}(D)=S$. By Lemmas 7.1 and 7.2, every $f \in A(S)$ induces an automorphism $f_{D}$ of $D$ such that $f_{S} \eta_{0}=\eta_{0} \circ f_{S}$, where $f_{S}$ denotes the automorphism of $S$ corresponding to $f$. It is well known that $A(S)$ acts on $S$ transitively. Since $D_{0}=\eta_{0}^{-1}\left(\sqrt{-1} v_{s}, 0\right)$, we have

$$
A(S) \cdot h_{0}\left(D_{0}\right)=h_{0}(D)
$$

We define a $G_{c}$-equivariant holomorphic diffeomorphism $\tilde{o}$ of $G_{c} / B_{0}$ onto $G_{c} / B$ by

$$
G_{c} / B_{0} \ni g B_{0} \stackrel{\tilde{\delta}}{\longrightarrow} g \delta^{-1} B \in G_{c} / B \text {. }
$$


Considering (6.10), we get the following diagram:

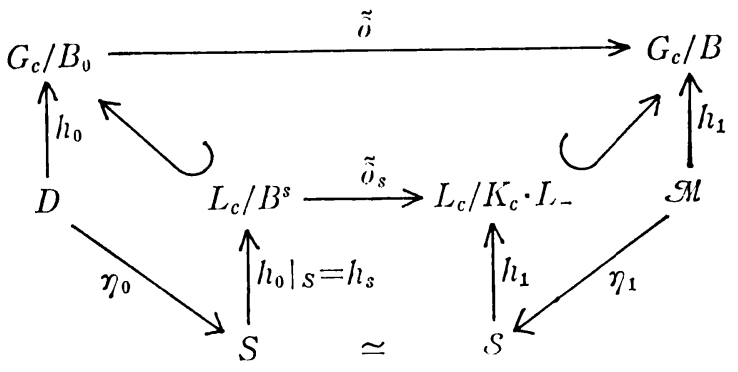

Lemma 7.3. $\tilde{\delta} \circ h_{0}\left(D_{0}\right)=h_{1}\left(\mathscr{M}_{0}\right)$.

Proof. Let $u=\left(\sqrt{-1} v_{s}, z_{2}, z_{3}, 0, w_{2}, w_{3}\right)$. Then

$$
\begin{aligned}
\tilde{\delta} h_{0}(u) & =\pi_{1} \cdot \exp \left(-\sqrt{-1} v_{r}\right) \cdot \exp z_{3} \cdot \exp w_{2} \cdot \exp w_{3} \cdot \exp z_{2} \cdot \exp \sqrt{-1} v_{s} \cdot \delta_{s}^{-1} \\
& =\pi_{1} \cdot \exp \left(-\sqrt{-1} v_{r}\right) \cdot \exp z_{3} \cdot \exp w_{2} \cdot \exp w_{3} \cdot \exp z_{2} .
\end{aligned}
$$

Here we used the fact $\exp \sqrt{-1} v_{s} \delta_{s}^{-1} \in K_{c} \cdot L_{-}$. Notice that

$$
\text { Ad } \delta_{s} z_{2}=\frac{1}{2}\left(z_{2}+\sqrt{-1}\left[\hat{v}_{s}, z_{2}\right]\right)
$$

and

$$
\frac{1}{2}\left(z_{2}-\sqrt{-1}\left[\hat{v}_{s}, z_{2}\right]\right)=A d \delta_{s}\left(-\frac{\sqrt{-1}}{2}\left[\hat{v}_{s}, z_{2}\right]\right) \in A d \delta_{s} t_{c}^{1} .
$$

Hence by Lemma 6.2 ,

$$
\begin{aligned}
\exp z_{2}= & \exp \frac{1}{2}\left(z_{2}+\sqrt{-1}\left[\hat{v}_{s}, z_{2}\right]\right) \cdot \exp \frac{1}{2}\left(z_{2}-\sqrt{-1}\left[\hat{v}_{s}, z_{2}\right]\right) \\
& \times \exp \frac{1}{8}\left[z_{2}-\sqrt{-1}\left[\hat{v}_{s}, z_{2}\right], z_{2}+\sqrt{-1}\left[\hat{v}_{s}, z_{2}\right]\right] \\
\equiv & \exp \left(A d \delta_{s} z_{2}\right) \cdot \exp \frac{\sqrt{-1}}{4}\left[z_{2},\left[\hat{v}_{s}, z_{2}\right]\right] \quad(\bmod B) .
\end{aligned}
$$

From the above equalities, we get $\tilde{\delta} \circ h_{0}(u)=h_{1}\left(u^{\prime}\right)$ for some $u^{\prime} \in \mathfrak{a}_{c}^{-2} \times\left(\mathfrak{a}_{+}+\mathfrak{t}_{+}\right)$. If we write $u^{\prime}=\left(z^{\prime}, w^{\prime}\right)$, then

$$
\begin{aligned}
& z^{\prime}=-\sqrt{-1} v_{r}+z_{3}+\frac{\sqrt{-1}}{4}\left[z_{2},\left[\hat{v}_{s}, z_{2}\right]\right] \\
& w^{\prime}=w_{2}+A d \delta_{s} z_{2}+w_{3} .
\end{aligned}
$$

It is not difficult to see that conversely for any $u^{\prime} \in \mathfrak{a}_{c}^{-2} \times\left(\mathfrak{a}_{+}+t_{+}\right)$, there exists $u$ of the form $u=\left(\sqrt{-1} v_{s}, z_{2}, z_{3}, 0, w_{2} w_{3}\right)$ such that $\tilde{\delta} \circ h_{0}(u)=h_{1}\left(u^{\prime}\right)$. Note that $\left[\bar{w}_{2}, \operatorname{Ad} \delta_{s} z_{2}\right]=\left[w_{2}, \overline{A d \delta_{s} z_{2}}\right]=0$ by Lemma 6.2. Using this equation and using Ad $\delta_{s} z_{2}=\frac{1}{2}\left[z_{2}+\sqrt{-1}\left[\hat{v}_{s}, z_{2}\right]\right.$ ), we get from (7.3),

$$
\operatorname{Im} z^{\prime}-F_{r}\left(w^{\prime}, w^{\prime}\right)+v_{r}=\operatorname{Im} z_{3}-\frac{1}{2}\left[\left[\operatorname{Im} z_{2}, \hat{v}_{s}\right], \operatorname{Im} z_{2}\right]-F\left(u_{2}^{\prime}+w_{3}, w_{2}+w_{3}\right) .
$$


This shows $u \in D_{0}$ if and only if $u^{\prime} \in \mathscr{M}_{0}$.

q.e.d.

Since $A(S)$ acts transitively on $S$, so on $\mathcal{S}$. It follows from Proposition 4.3, $h_{1}(\mathscr{M})=A(S) \cdot h_{1}\left(\mathscr{M}_{0}\right)$. Since the mapping $\tilde{\delta}$ commutes with the action of $A(S)$, considering the diagram (7.2), we get by (7.1) and Lemma 7.3,

$$
\tilde{\delta} \circ h_{0}(D)=h_{1}(\mathscr{M}) \text {. }
$$

We thereby proved that $D$ is holomorphically isomorphic to $\mu$. In particular, $D$ is connected and so is $\Omega$ (cf. Proof of Proposition 5.6). Let $w=w_{1}+w_{2}+w_{3}$ and suppose $F(w, w)=0$, where $w_{1} \in P\left(\mathfrak{g}^{-1}\right), w_{2} \in t_{+} \cap t_{c}^{0}$ and $w_{2} \in \mathfrak{a}_{+}$. Then $F_{s}\left(w_{1}, w_{1}\right)$ $=0$. Since $F_{s}$ is an $\Omega_{s}$-hermitian form, we have $w_{1}=0$. Hence $F(w, w)=F_{r}(w, w)$. Therefore $w_{2}=w_{3}=0$. We have proved the following

Theorem 7.4. Let $M$ be half-homogeneous hyperbolic manifold satisfying the condition $(C)$ in $\S 3$. Then $M$ is immersed in a complex vector space as a pseudoSiegel domain $D$ of the second kind in such a way that $G(M)$ acts on $D$ equivariantly.

\section{§8. Characterizations of Siegel domains.}

Let $D$ be a pseudo-Siegel domain of the second kind associated with a connected cone $\Omega$ in a real vector space $V$ and a $V_{c}$-valued hermitian form $F$ on $W$. We prove

Proposition 8.1. A pseudo-Siegel domain $D$ of the second kind is a Siegel domain of the second kind if and only if $D$ is a complete hyperbolic manifold.

Proof. Assume that $D$ is complete hyperbolic. Let $(z, w) \in D$, where $z \in V_{c}$ and $w \in W$. Then $(\sqrt{-1}(\operatorname{Im} z-F(w, w)), 0) \in D$. Therefore if we set $D_{1}=D \cap\left(V_{c} \times\{0\}\right)$, then $D_{1} \neq \phi$ and $D_{1} \cong\left\{z \in V_{c} ; \operatorname{Im} z \in \Omega\right\}$. By Theorem 3.4, Ch. $\mathrm{V}$ of [3], $D$ is holomorphically convex. We then have from the proof of Proposition 1.1 in [13],

(a) $(z, w) \in D$ implies $\in(z, 0) \in D_{1}$.

(b) $\Omega$ is convex.

Suppose that $\Omega$ contains a line. Then $D_{1}$ contains a complex line which contradicts the assumption that $D$ is hyperbolic. Therefore $\Omega$ is a convex cone containing no entire straight lines. For any $v \in \Omega$ and $w \in W$, we know $(\sqrt{-1}(v+F(w, w)), w) \in D$. Therefore from (a), $v+F(w, w) \in \Omega$. Since $v$ is arbitrary, $F(w, w)$ is in the closure of $\Omega$. This implies $F$ is an $\Omega$-hermitian form. Hence $D$ is a Siegel domain of the second kind. The converse follows from Theorem 4.15, Ch. IV of [3]. q.e.d.

As an immediate consequence of Theorem 7.4 and Proposition 8.1, we have

Theorem 8.2. Let $M$ be a half-homogeneous hyperbolic manifold. Assume 
the followings:

(a) $M$ is complete hyperbolic.

(b) $M$ satisfies the condition $(C)$ in $\S 3$.

(c) $\mathfrak{b}^{0}(p) \neq \mathfrak{b}^{0}(q)$ if $p \neq q$.

Then $M$ is holomorphically isomorphic to a Siegel domain of the second kind. Conversely, every Siegel domain of the second kind is a half-nomogeneous hyperbolic manifold satisfying (a), (b) and (c).

The converse follows from Theorem 4.15, Ch. IV of [3], Corollary 5 of [8] and from Proposition 6.2 of [9].

In the case where $M$ is a homogeneous hyperbolic manifold satisfying $(C)$, the equivariant immersion $\Phi$ becomes a covering mapping. Then by Theorem 4.7, Ch. IV of [3], the corresponding pseudo-Siegel domain $D$ is complete hyperbolic. Applying Proposition 8.1, $D$ becomes a homogeneous Siegel domain of the second kind. In particular, $D$ is simply connected. Consequently, $\Phi$ is a holomorphic diffeomorphism of $M$ onto $\mathcal{N}$. Therefore $M$ is holomorphically isomorphic to $D$ and hence isomorphic to a homogeneous bounded domain. Thus we obtain the following

Theorem 8.3. A homogeneous bounded domain in $\boldsymbol{C}^{n}$ is a homogeneous hyperbolic manifold satisfying the condition $(C)$ in $\S 3$.

\section{Department of Mathematics, KYOTO UNIVERSITY}

\section{References}

[1] S. Helgason, Differential Geometry, Lie groups, and Symmetric spaces, Acad. Press, New York, San Francisco, London, 1978.

[2] W. Kaup, Y. Matsushima and T. Ochiai, On the automorphisms and equivalences of generalized Siegel domains, Amer. J. Math., 92 (1970), 475-498.

[3] S. Kobayashi, Hyperbolic Manifolds and Holomorphic Mappings, Marcel Dekker, New York, 1970.

[4] S. Kobayashi, Transformation Groups in Differential Geometry, Springer Verlag, Berlin, Heidelberg, New York, 1972.

[5] A. Kodama and H. Shima, Characterizations of homogeneous bounded domains, Tsukuba J. Math., 7 (1983), 79-86.

[6] K. Nakajima, Symmetric spaces associated with Siegel domains, J. Math. Kyoto Univ., 15 (1975), 303-349.

[7] K. Nakajima, On realization of Siegel domains of the second kind as those of the third kind, J. Math. Kyoto Univ., 16 (1976), 143-166.

[8] K. Nakajima, On equivariant holomorphic imbeddings of Siegel domains to compact complex homogeneous spaces, J. Math. Kyoto Univ., 19 (1979), 471-480.

[9] K. Nakajima, On certain hermitian symmetric submanifolds of bounded domains, J. Math. Kyoto Univ., 23 (1983), 409-425.

[10] K. Nakajima and Y. Se-ashi, On totally geodesic hermitian symmetric submanifolds of Kähler manifolds, Proc. Japan Acad., 58 (1982), 163-166.

[11] I.I. Pyatetski-Shapiro, Géométrie des domains classiques et théorie des functions 
automorphes, Dunod, Paris, 1966.

[12] N. Tanaka, On infinitesimal aułomorphisms of Siegel domains, J. Math. Soc. Japan, 22 (1970), 180-212.

[13] J. Vey, Sur la division des domains de Siegel, Ann. Sci. École Norm. Sup., 4 Série, 3 (1971), 479-506. 